\title{
Nuclei from lattice QCD
}

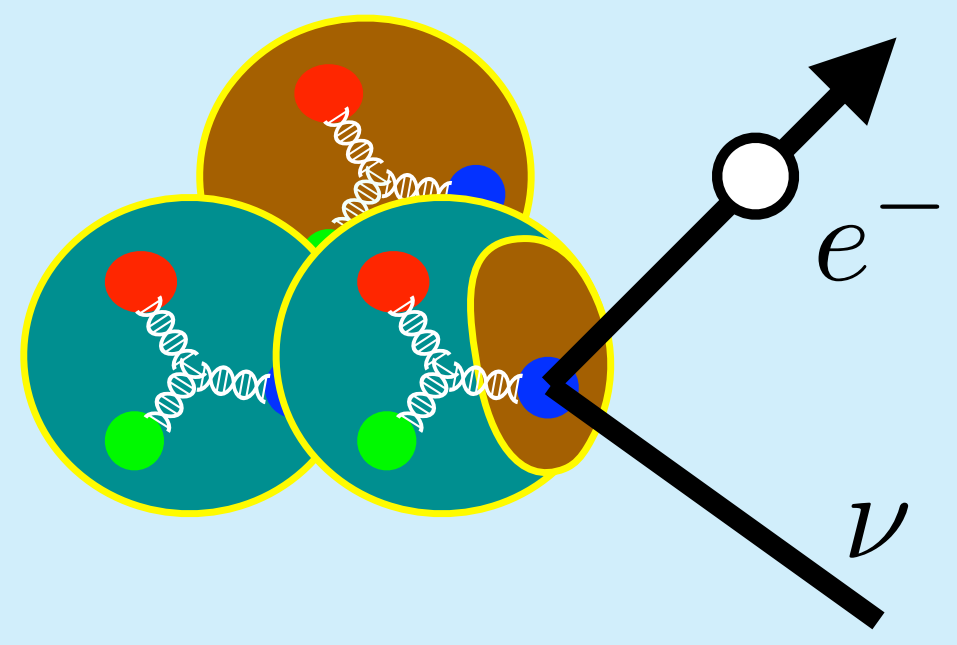

Michael Wagman

APS April Meeting 2020: From Quarks to the Cosmos

April, 202020

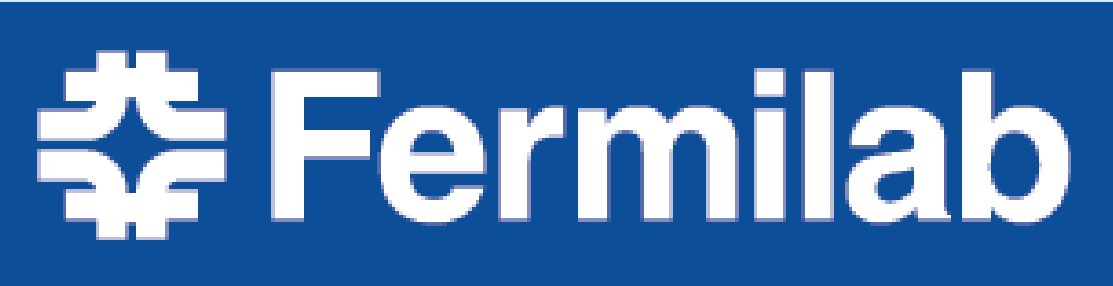




\section{Nuclear models}

Nucleon-nucleon scattering can be approximately described by a non-relativistic two-body potential
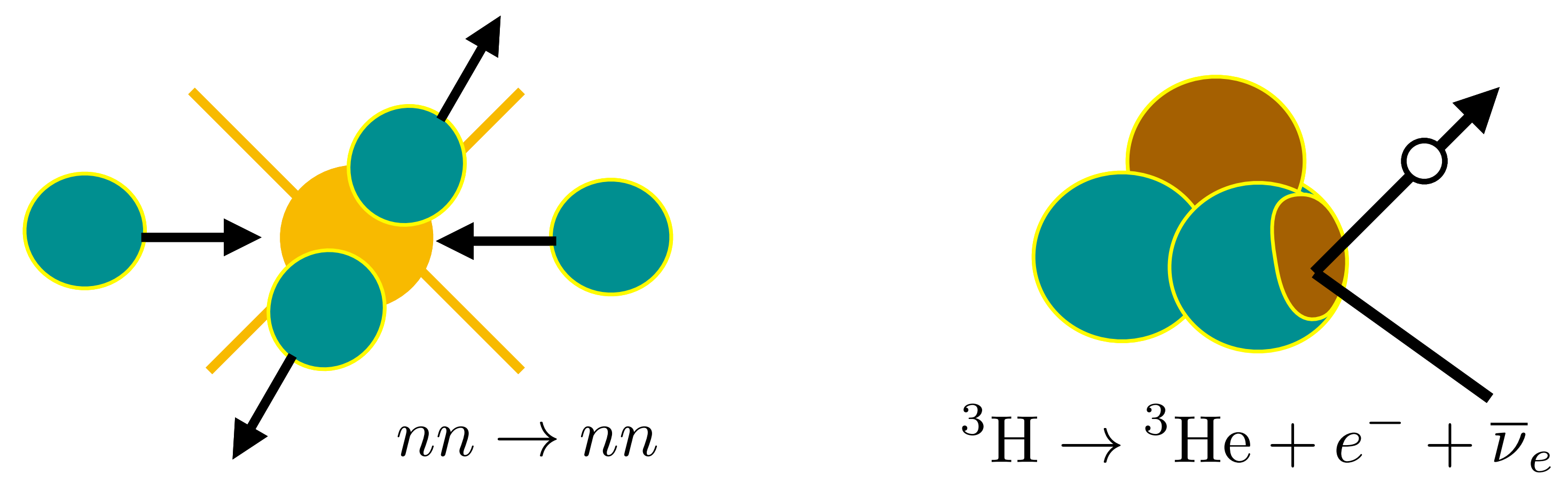

Fundamentally, this description is incomplete 


\section{Nuclear models}

Nuclear scattering and reaction rates can be approximately described using a two-nucleon potential and one-nucleon currents
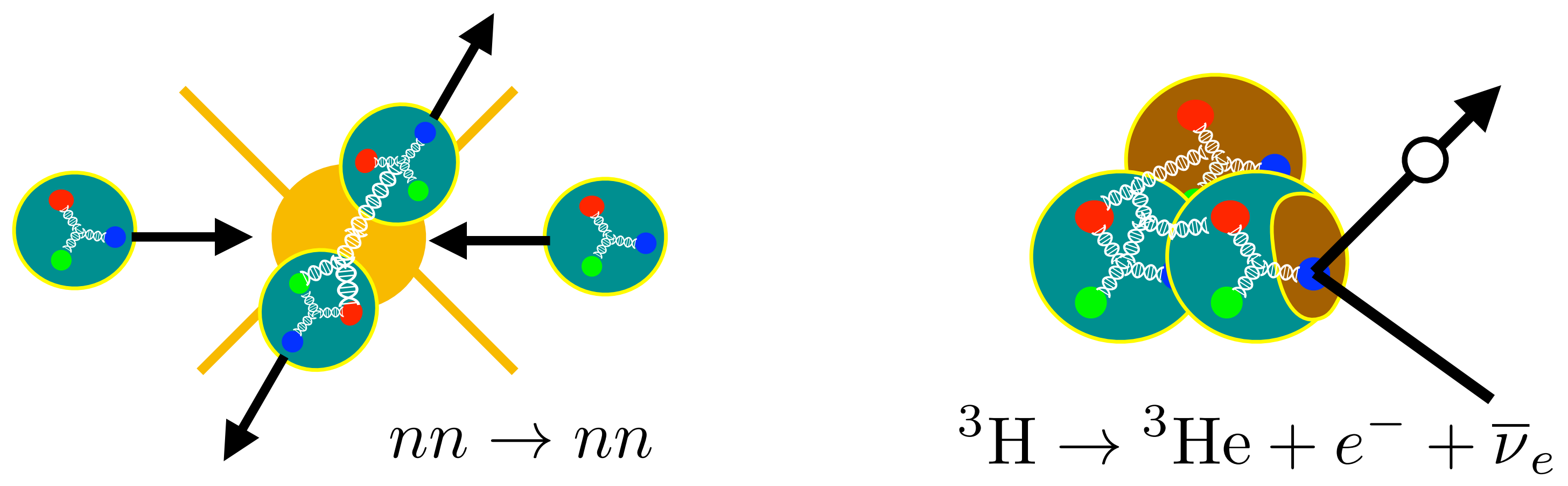

Fundamentally, this description is incomplete

Protons and neutrons are composite objects made of quarks and gluons

Nuclear processes affected by correlations:

- Three-nucleon forces

- Two-nucleon currents
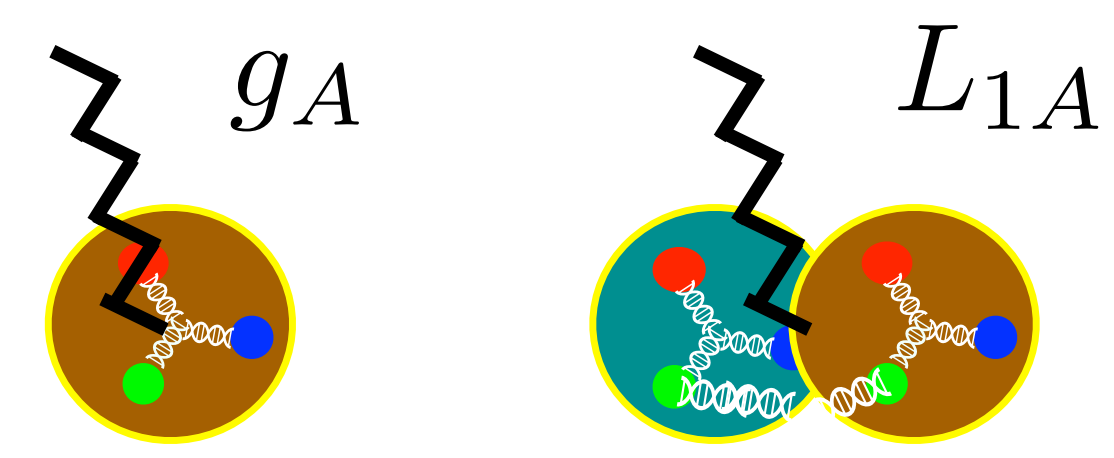


\section{Nuclei, neutrinos, and new physics}

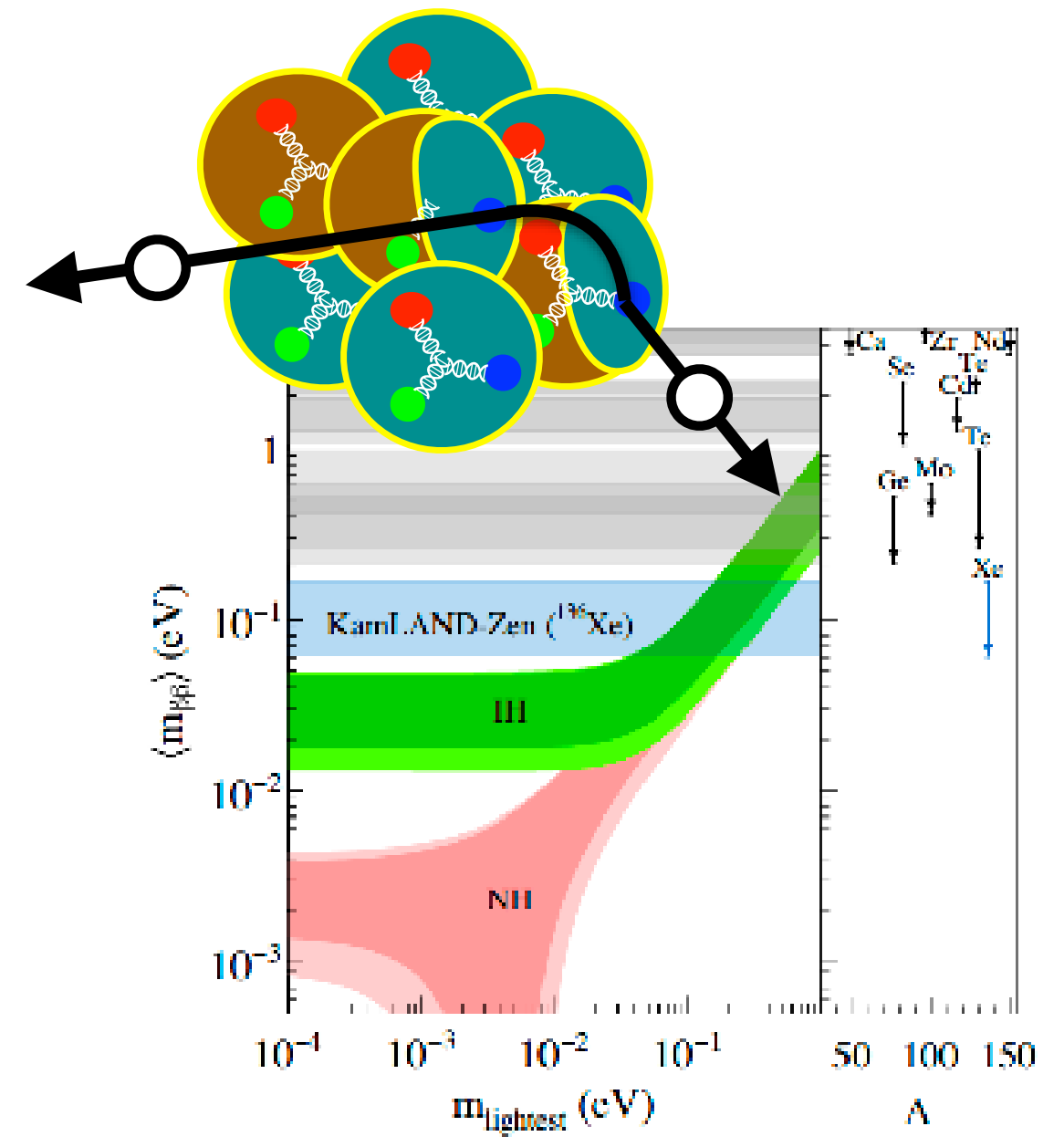

Many experiments are searching for new physics using nuclear targets

- Dark matter, fundamental symmetry violation, ...

Accurate theoretical predictions are needed to reliably interpret new physics searches

Electroweak reaction cross-sections critical for neutrino oscillation experiments, $0 \nu \beta \beta$ searches

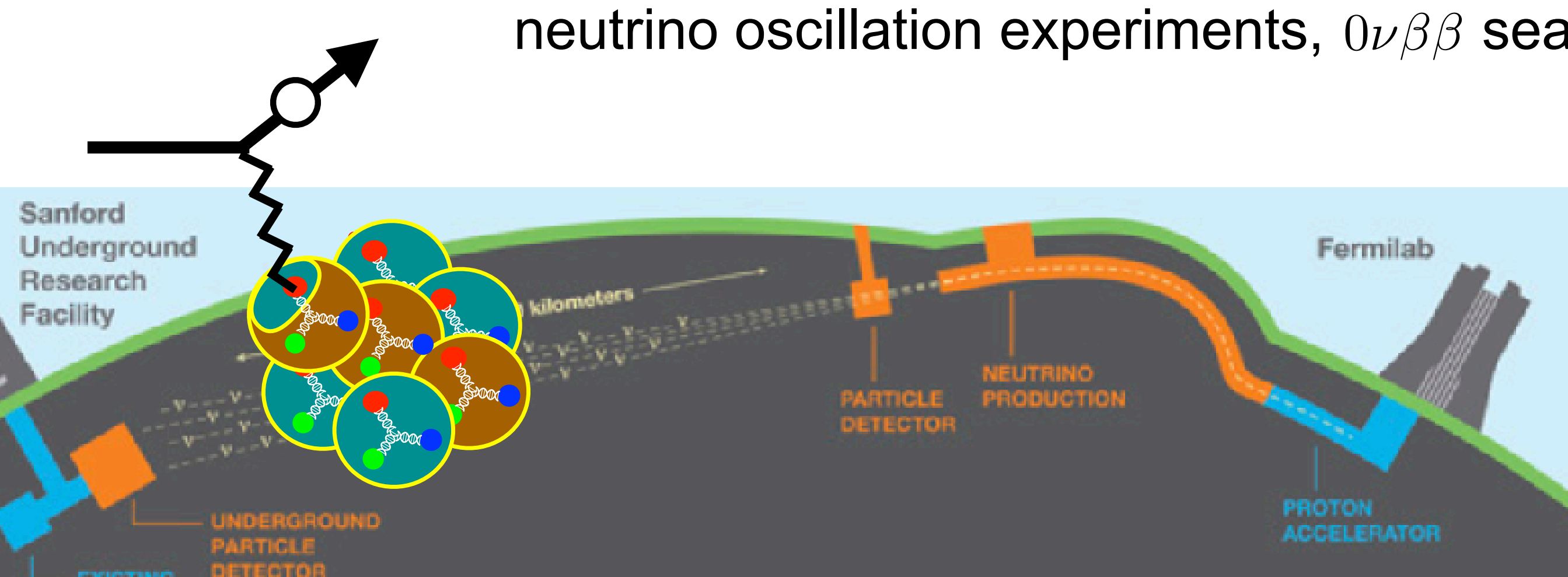




\section{QCD and DUNE}

Neutrino-nucleus cross-section needed from theory (QCD + leptons) for DUNE to determine neutrino mass hierarchy and $C P$ violation

Oscillation parameters determined by comparing reconstructed spectrum at far detector vs predictions from near detector + theory

Neutrino energy reconstruction with at least $100 \mathrm{MeV}$ accuracy needed to observe $C P$ violation

Mosel Ann. Rev. Nucl. Part. Sci. 66, (2016)

Abi et al (DUNE), arXiv 1807.10334

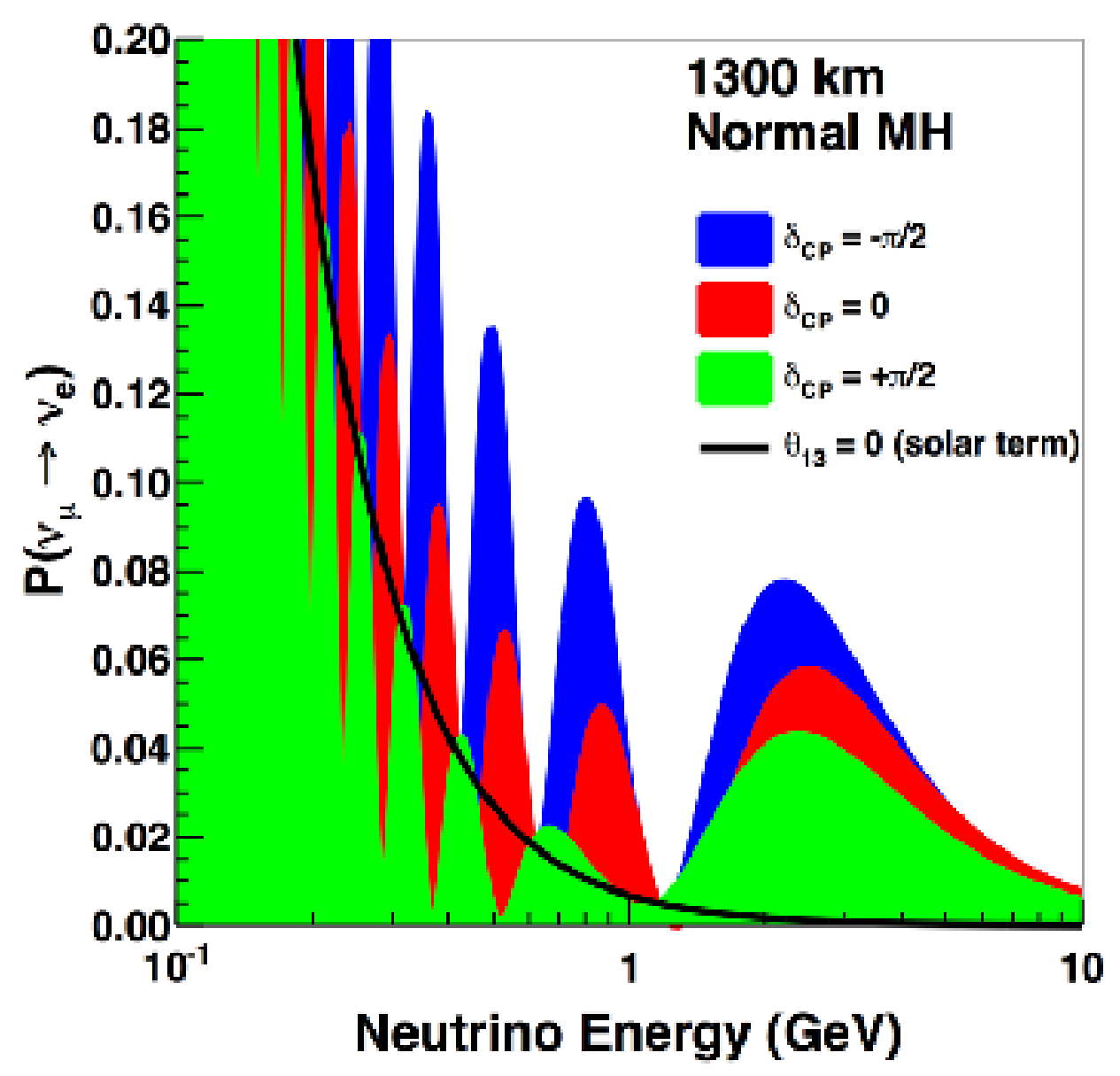

Acciarri et al (DUNE) arXiv 1512.06148 


\section{Double $\beta$-decay}

Doubly-weak reactions important but less well-understood phenomenologically

Double-beta decay reactions include additional two-body currents not present in single-beta decay, e.g. isotensor axial polarizability for $2 \nu \beta \beta$

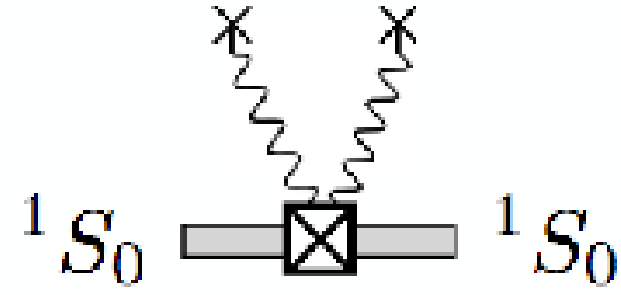

Shanahan, MW et al [NPLQCD], PRL 119 (2017)

Tiburzi, MW et al [NPLQCD], PRD 96 (2017)

Two-body currents needed for $0 \nu \beta \beta$ at leading order in ChEFT

Cirigliano et al, PRL 120 (2018)

LQCD studies of two-nucleon systems can be used to fix unknown LEC

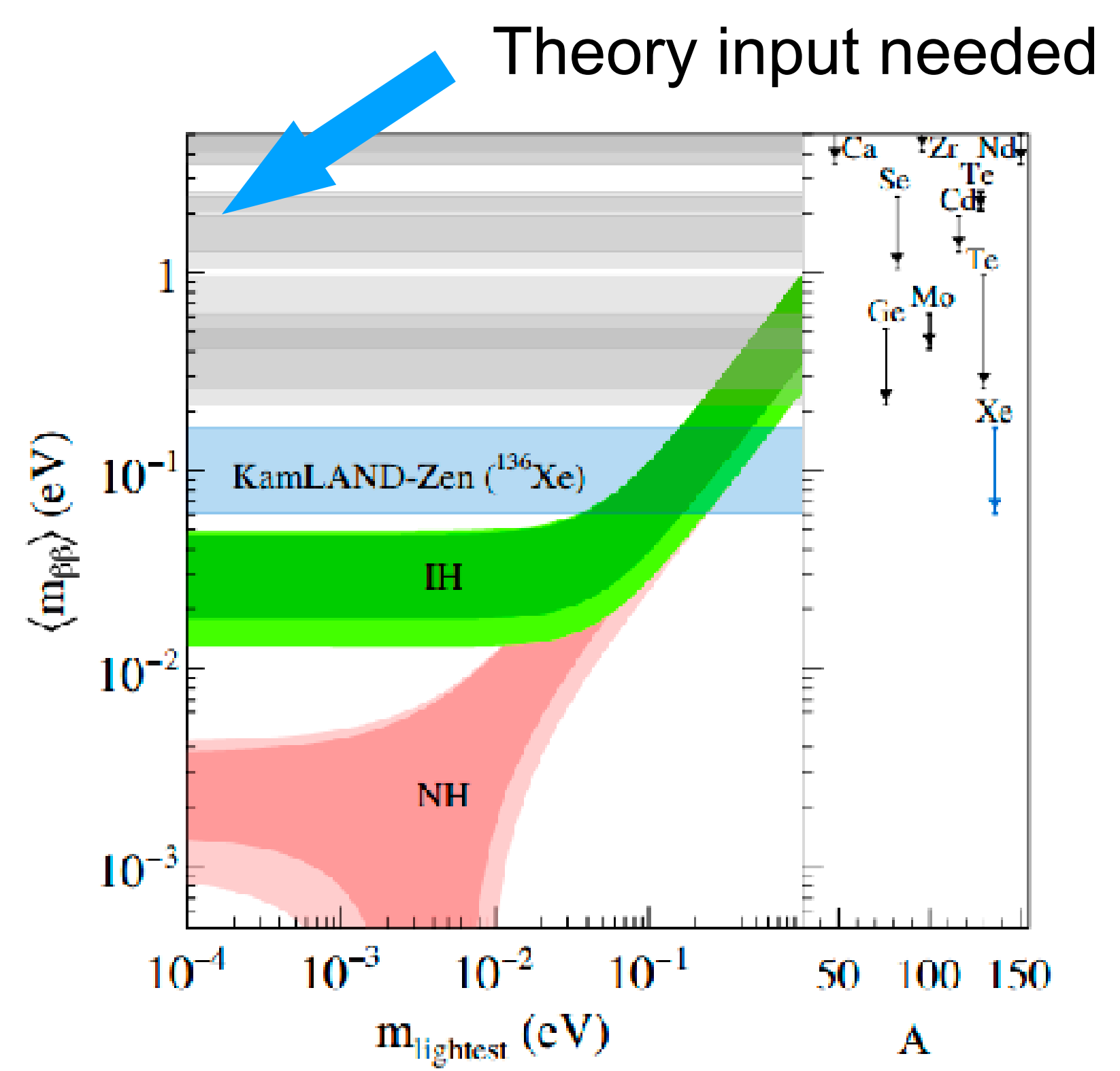




\section{From quarks to nuclei}
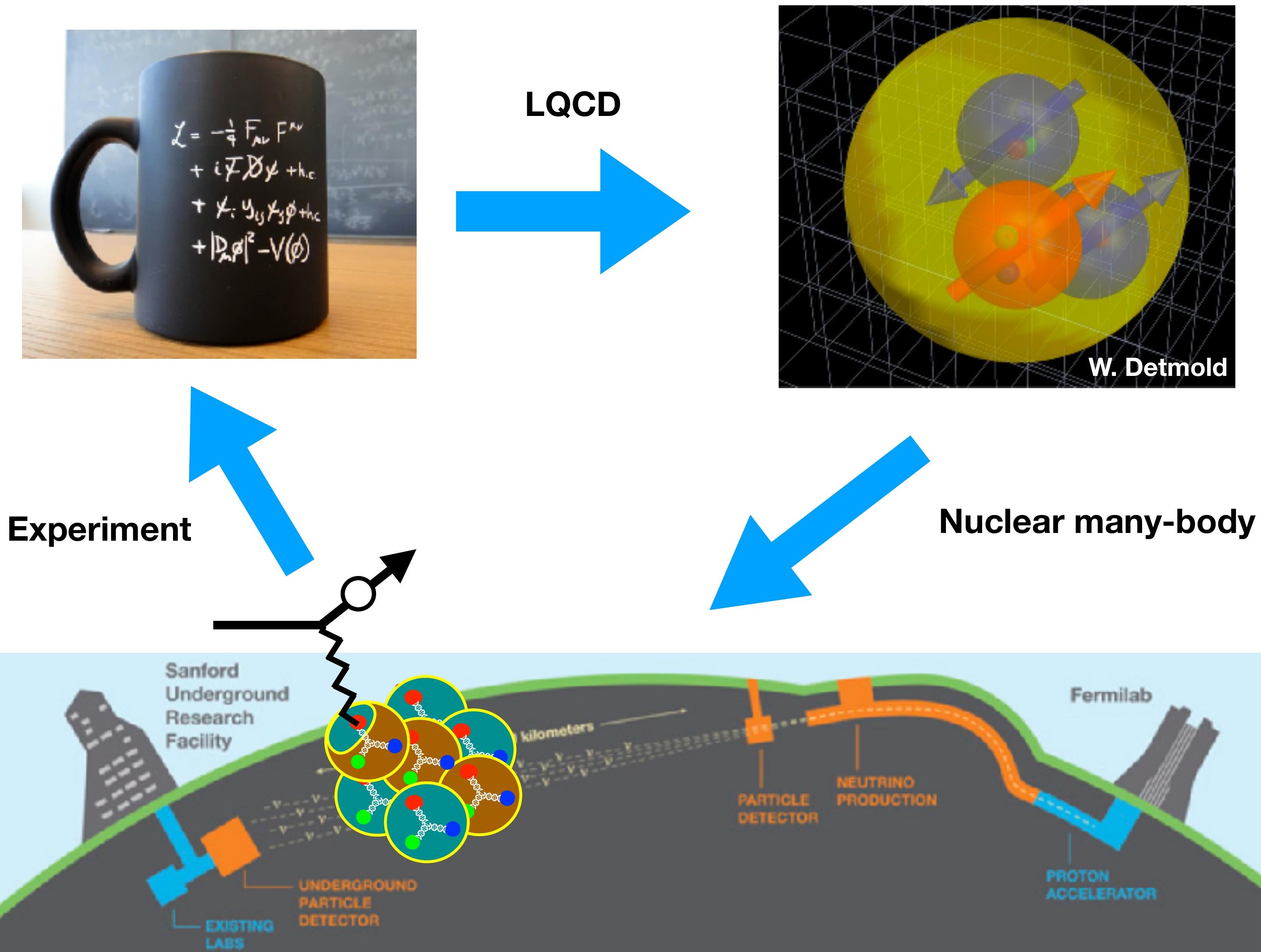


\section{Lattice QCD}

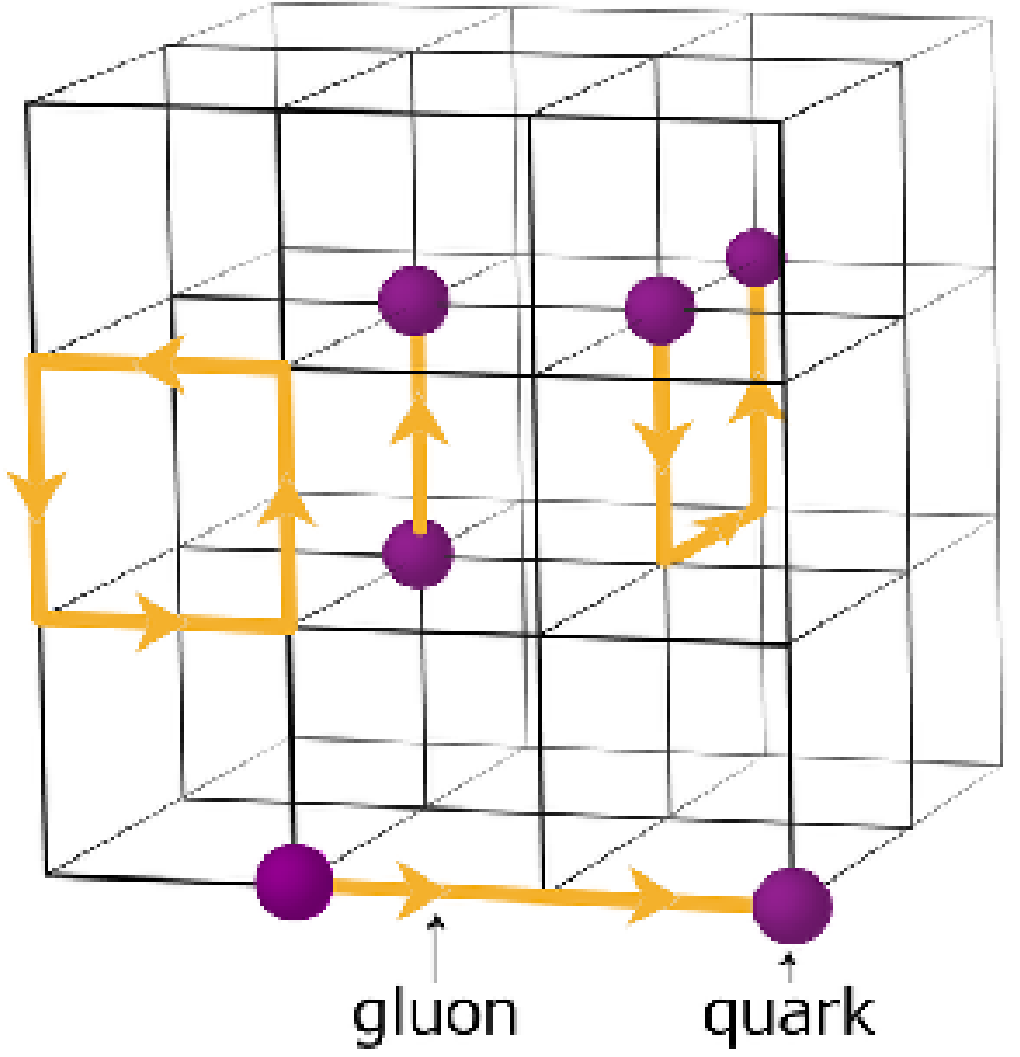

Lattice action defines an EFT for QCD valid at lengths larger than lattice scale

QCD properties of hadrons reproduced by LQCD up to lattice cutoff artifacts removed by continuum extrapolation
Approximating spacetime as a discrete lattice with a finite size makes QCD path integrals finite

$$
\begin{aligned}
& \langle\mathcal{O}\rangle=Z^{-1} \int \mathcal{D} U \mathcal{D} \bar{q} \mathcal{D} q e^{-S_{Q C D}(U, q, \bar{q})} \mathcal{O}(U, q, \bar{q}) \\
& =Z^{-1} \int \mathcal{D} U e^{-S_{g}(U)} \operatorname{det}\left(\not D(U)+m_{q}\right) \mathcal{O}\left(U,\left[\not D(U)+m_{q}\right]^{-1}\right)
\end{aligned}
$$




\section{Hadron-hadron interactions}

Volume scaling of energy levels allows bound and scattering states to be distinguished using finite-volume Euclidean information

\section{Infinite-volume scattering state}

$$
[E(L)-E(\infty)] \propto \frac{a}{M L^{3}}
$$

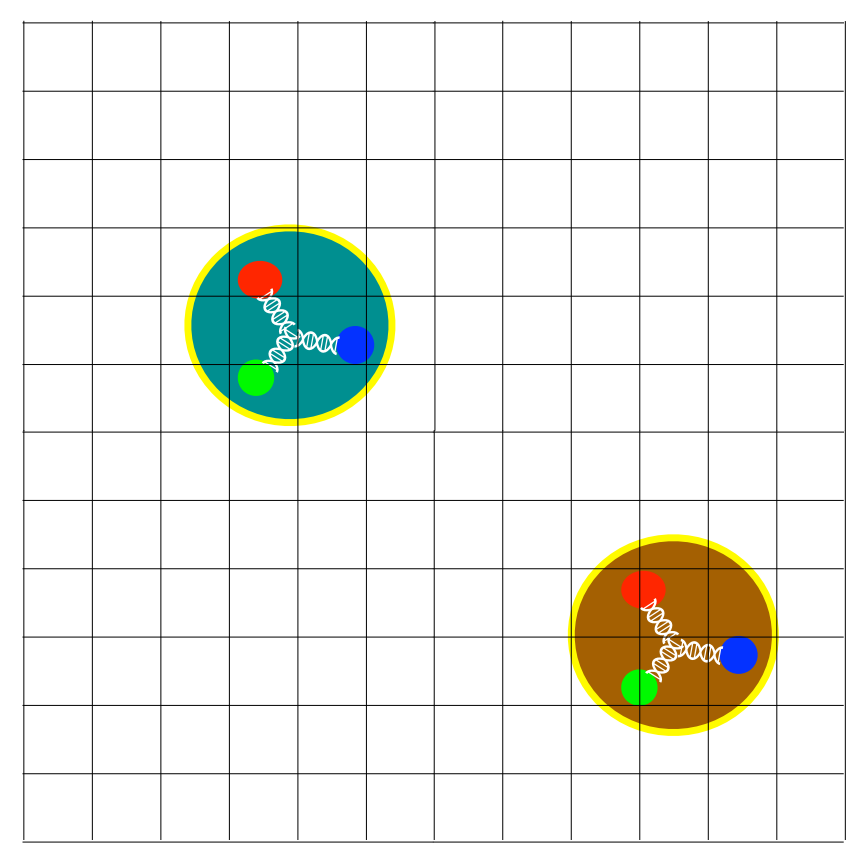

Quantitative information about interactions encoded in volume dependence

Lüscher, Commun. Math. Phys. 105 (1986)

\section{Infinite-volume} bound state

$$
[E(L)-E(\infty)] \propto \frac{e^{-\gamma L}}{L}
$$

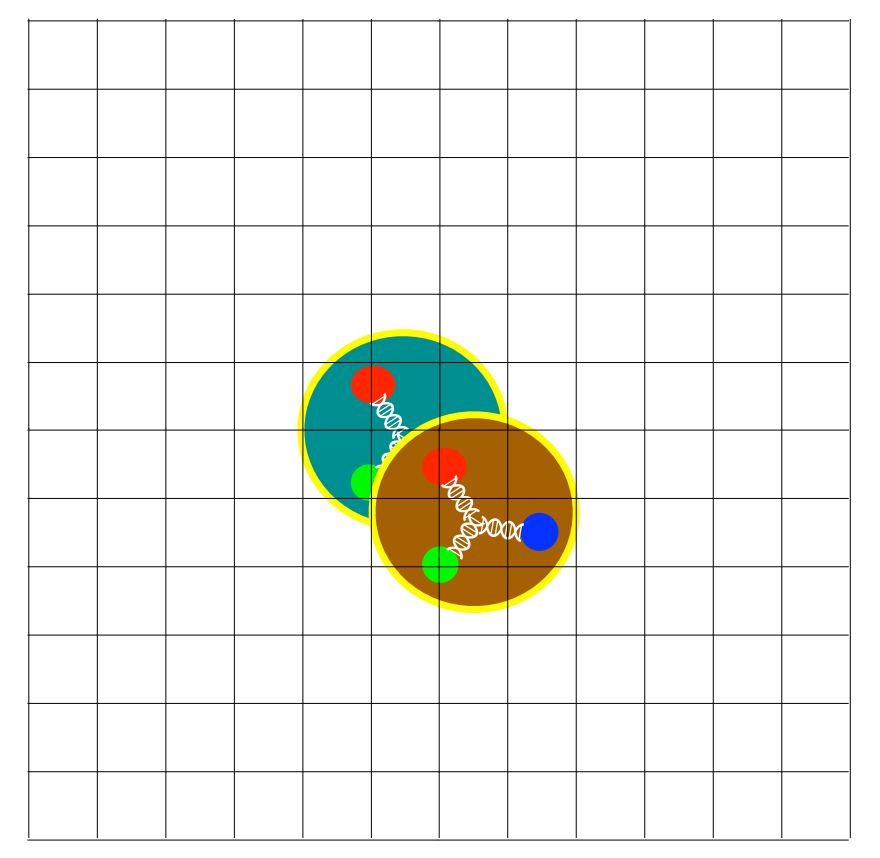

Beane et al, Phys. Lett. B 585 (2004)

Huang, Yang, Phys. Rev. 105 (1957) 


\section{Neutrino-Nucleus Scattering}

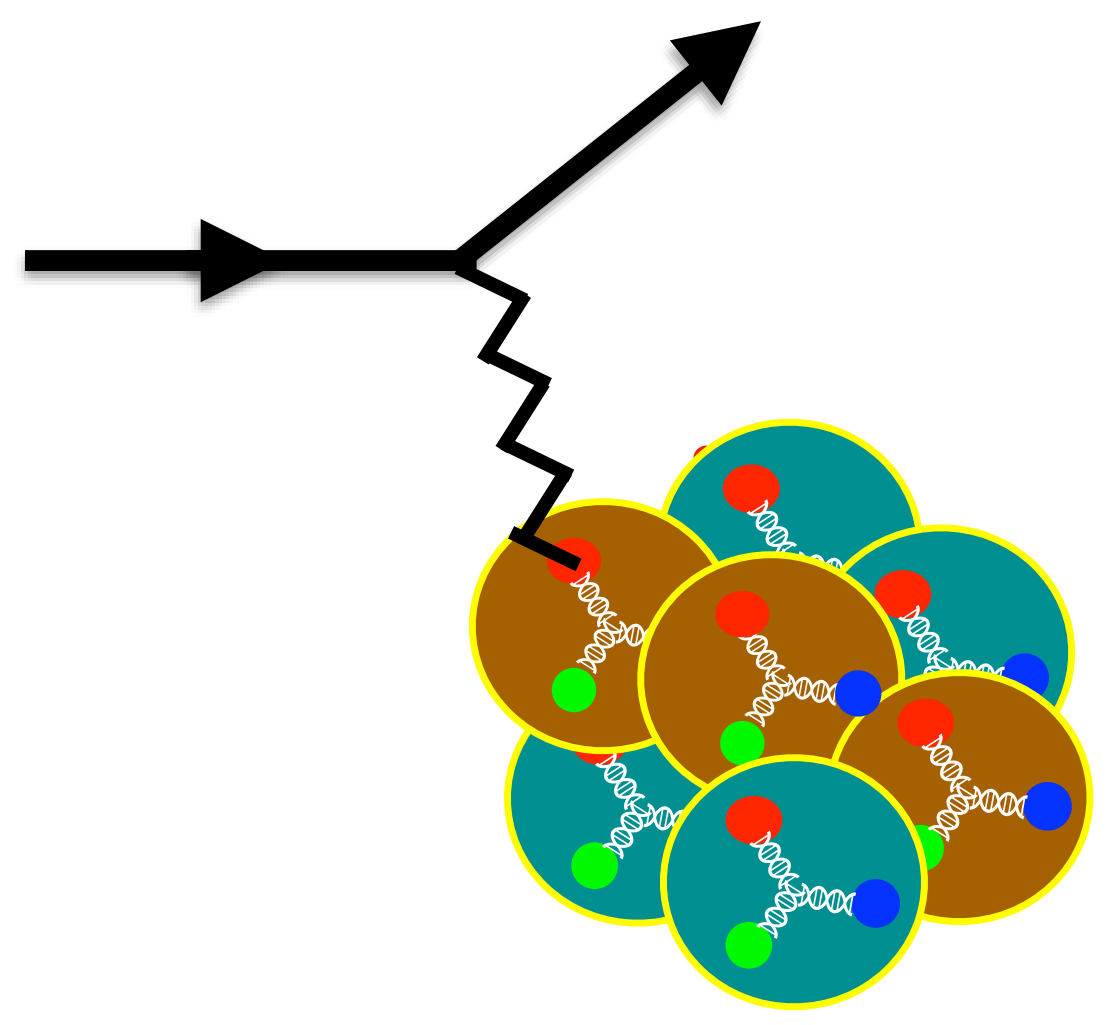

Single-nucleon response

dominates for small nuclei
Cross-section governed by vector and axial form factors of nuclei in QCD

Vector form factors can be measured in electron scattering experiments

Axial form factors, flavor dependence less well-known

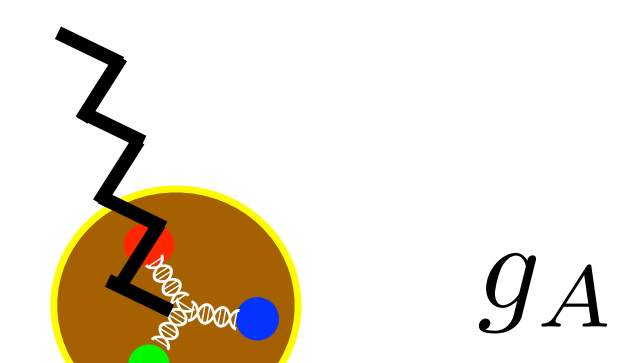

Multi-nucleon effects more important for larger nuclei 


\section{Single-Nucleon QCD Input}

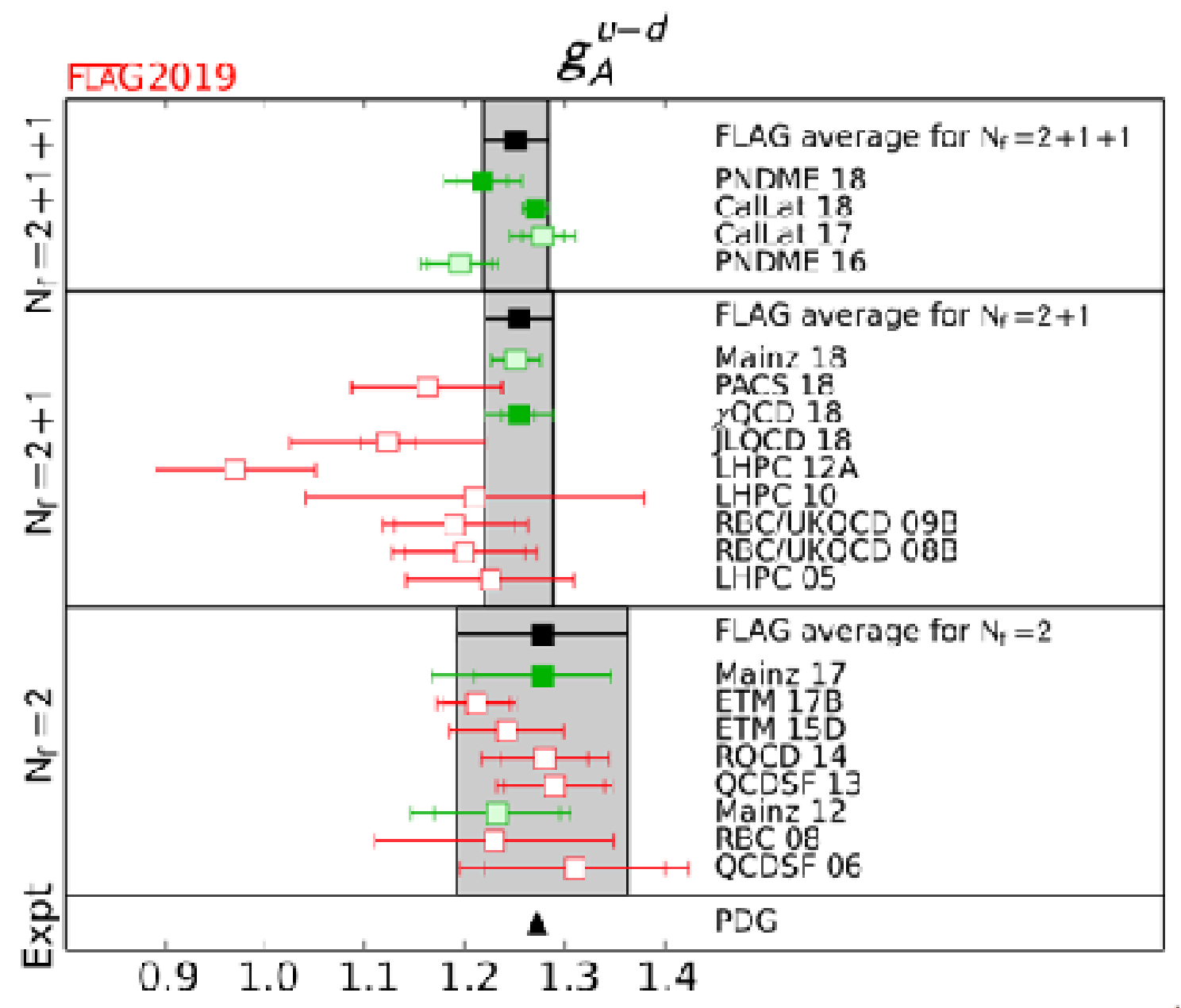

Nucleon axial charge computed in LQCD by several groups

Results (with controlled systematics) consistent with neutron decay experiments

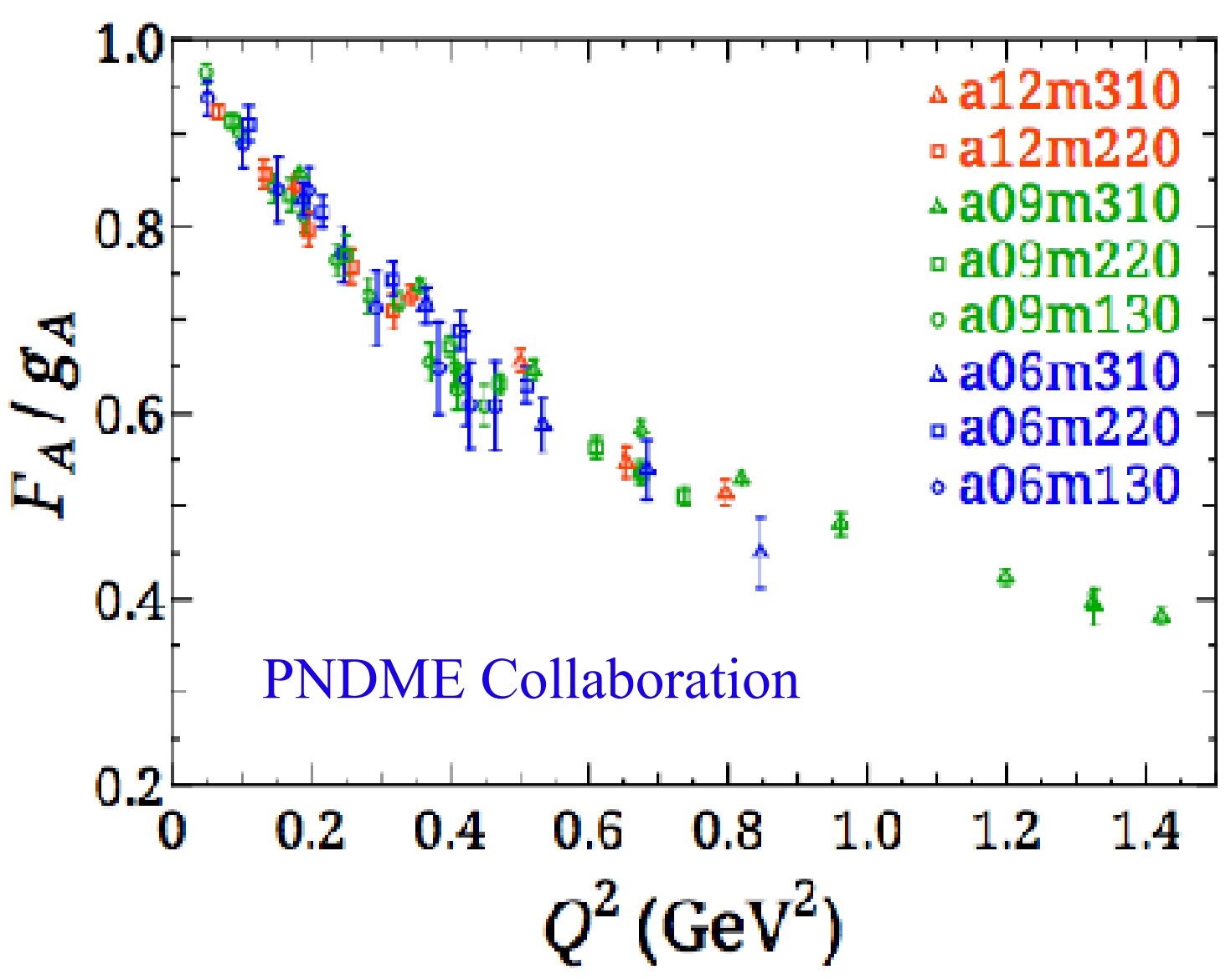

Kronfeld et al [USQCD], arXiv:1904.09931 


\section{Axial currents in nuclei}

Axial current matrix elements are smaller than predicted by shell models, experiment reproduced by rescaling $g_{A} \rightarrow q g_{A}$ with $q \sim 0.75$

Modern calculations including multinucleon correlations can reproduce experiment without "quenching" $g_{A}$
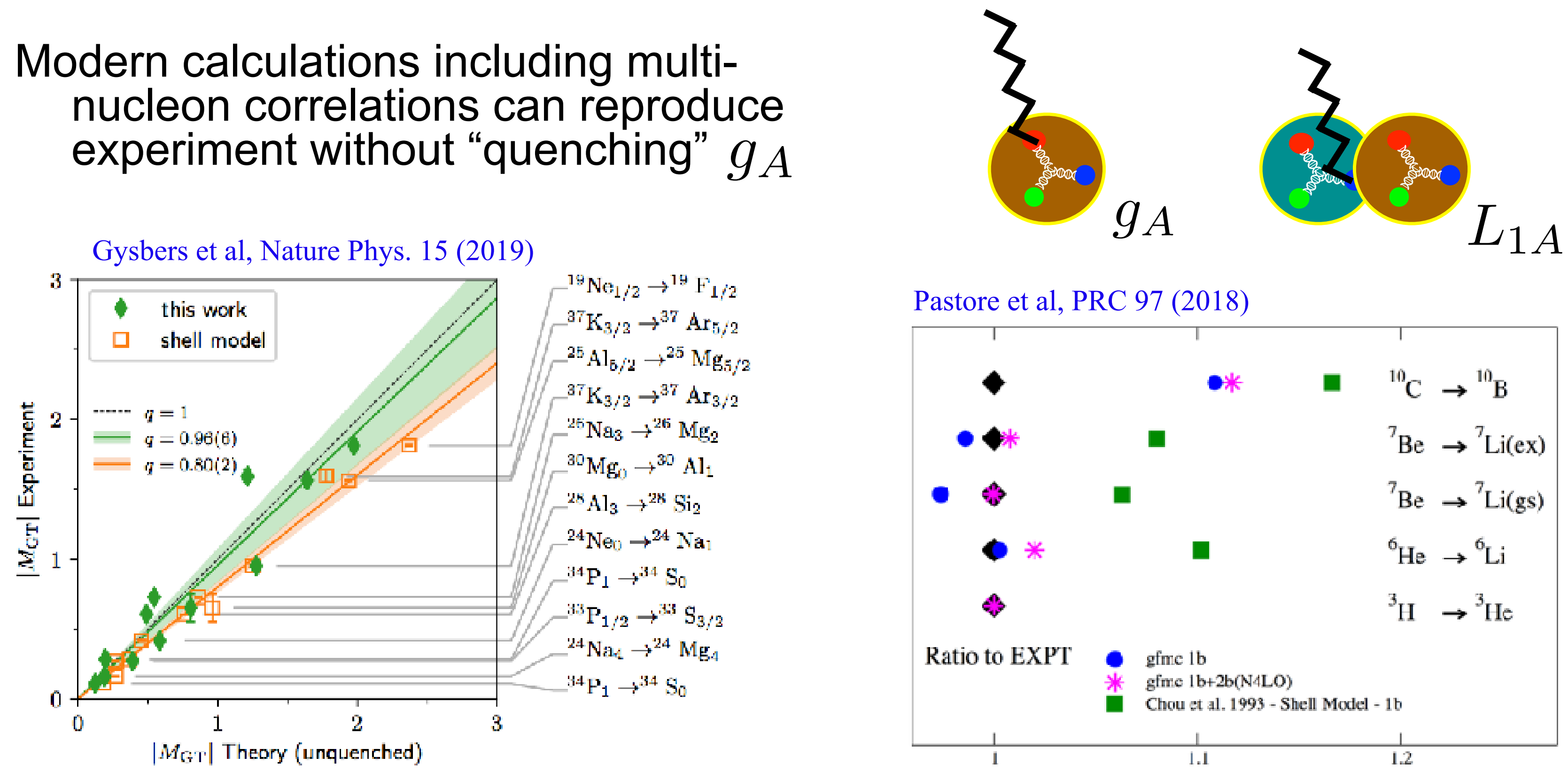

Pastore et al, PRC 97 (2018)

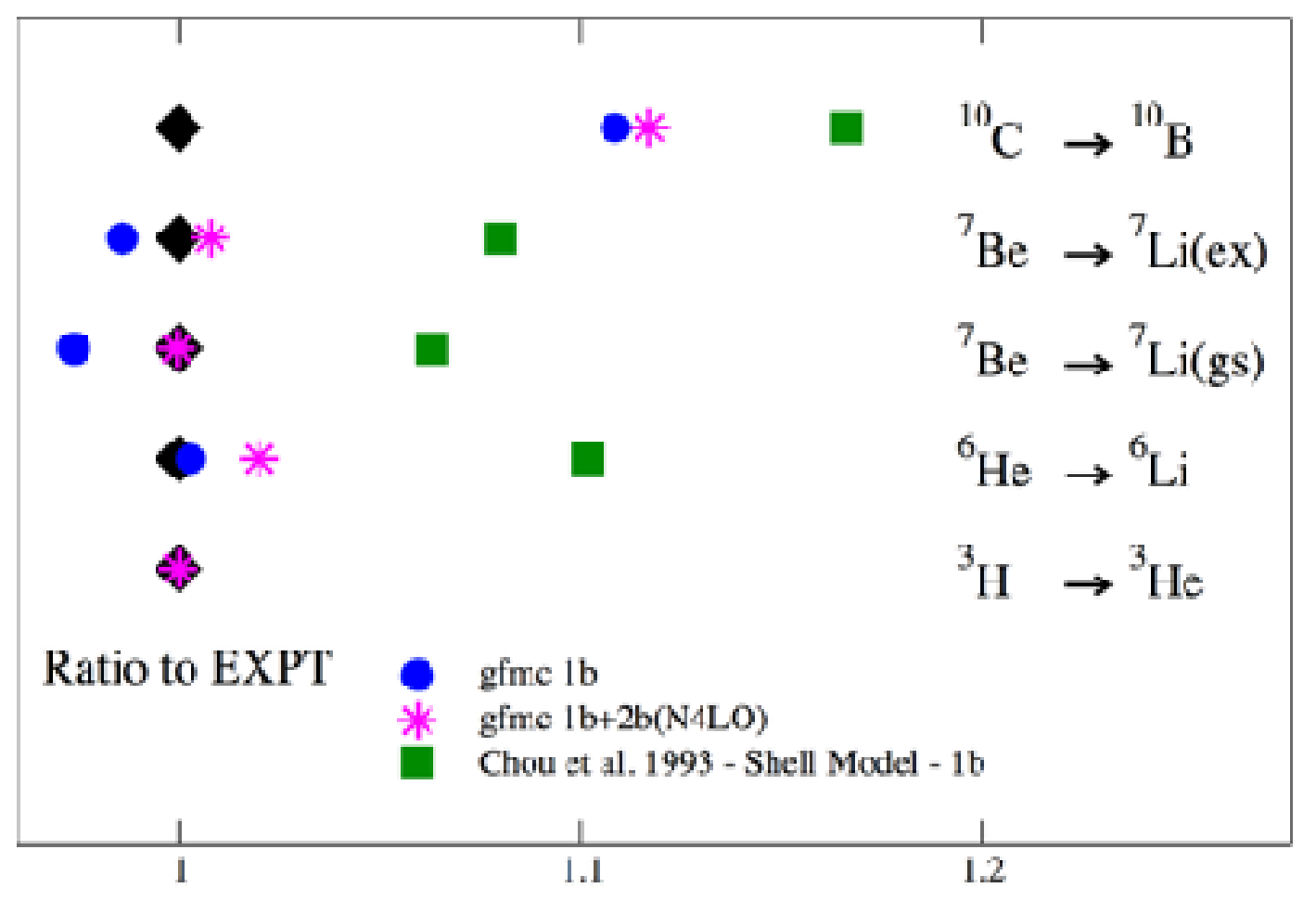

Can the emergence of nuclear effects on axial currents be predicted directly from the Standard Model? 


\section{Nuclei from LQCD}

LQCD can calculate spectra and matrix elements of QCD with IR cutoff (finite volume) and UV cutoff (lattice spacing) in Euclidean spacetime

Volume dependence encodes scattering phase shifts, binding energies determined by QCD spectrum in large volumes
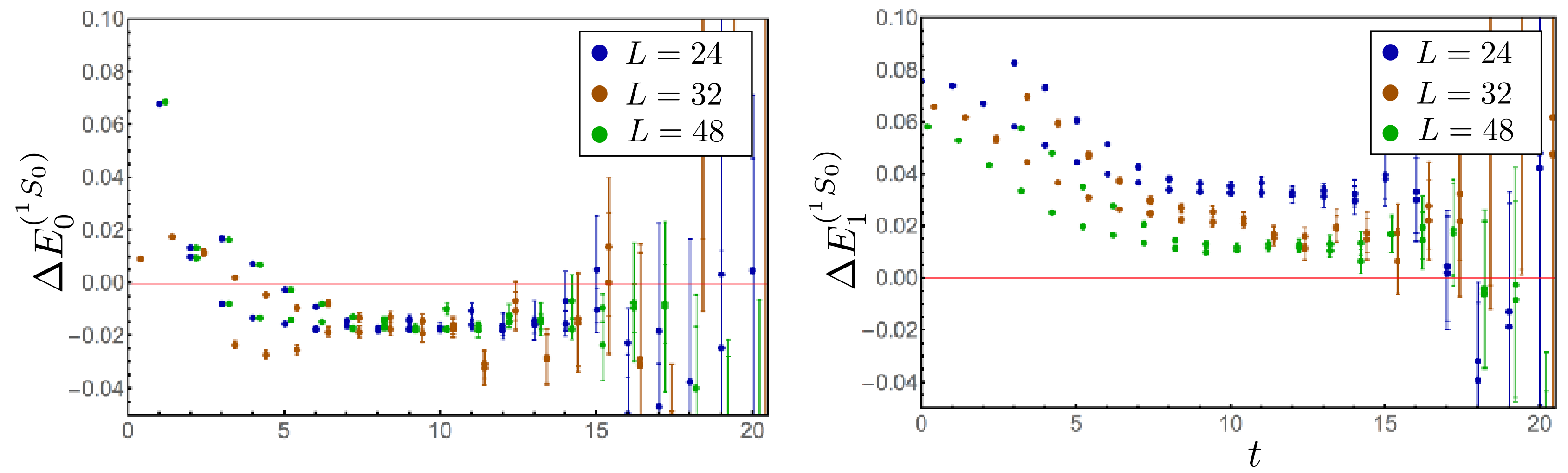

NPLQCD collaboration finds nuclei and hypernuclei with 2-5 baryons are bound at $m_{\pi} \sim 806 \mathrm{MeV}$ more deeply than in nature

Beane et al [NPLQCD], PRD 87 (2013) $\quad L=2.9 \mathrm{fm} \rightarrow 5.8 \mathrm{fm} \quad a=0.145 \mathrm{fm}$

PACS collaboration finds similar results at $m_{\pi} \sim 510 \mathrm{MeV}$

Yamazaki et al, PRD 86 (2012)

$$
L=3.5 \mathrm{fm} \rightarrow 7.0 \mathrm{fm} \quad a=0.09 \mathrm{fm}
$$




\section{Nuclei from LQCD}

LQCD can calculate spectra and matrix elements of QCD with IR cutoff (finite volume) and UV cutoff (lattice spacing) in Euclidean spacetime

Volume dependence encodes scattering phase shifts, binding energies determined by QCD spectrum in large volumes
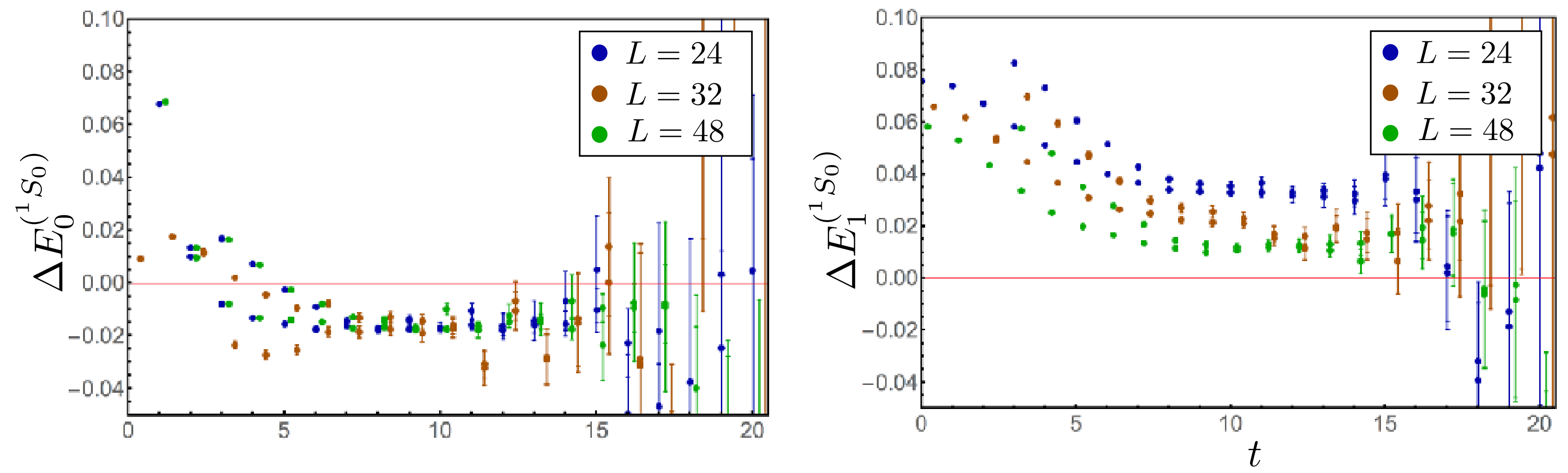

NPLQCD collaboration finds nuclei and hypernuclei with 2-5 baryons are bound at $m_{\pi} \sim 806 \mathrm{MeV}$ more deeply than in nature

Beane et al [NPLQCD], PRD 87 (2013) $\quad L=2.9 \mathrm{fm} \rightarrow 5.8 \mathrm{fm} \quad a=0.145 \mathrm{fm}$

PACS collaboration finds similar results at $m_{\pi} \sim 510 \mathrm{MeV}$

Yamazaki et al, PRD 86 (2012)

$$
L=3.5 \mathrm{fm} \rightarrow 7.0 \mathrm{fm} \quad a=0.09 \mathrm{fm}
$$




\section{Nuclear matrix elements}

LQCD spectrum determined from 2-point correlation functions

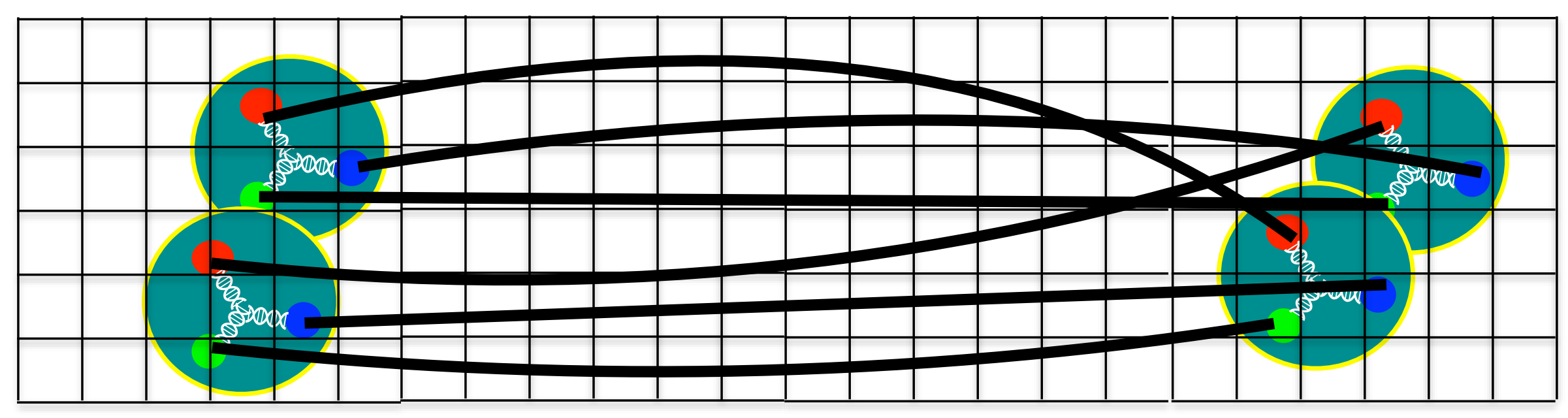

$$
C_{A}(t)=<A(t) A^{\dagger}(0)>=\sum_{n}\left|Z_{n}\right|^{2} e^{-E_{n} t}
$$

Axial matrix elements determined from 3-point correlation functions including an axial current operator insertion

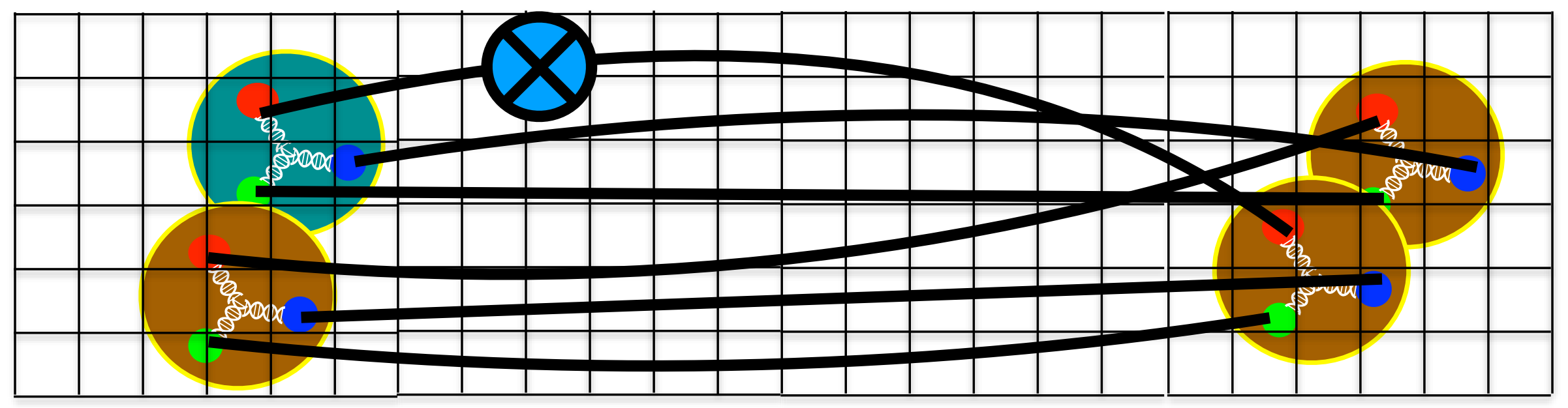




\section{Fixed-order background fields}

Basic input: linear combinations of quark propagators with propagators that contain a current inserted everywhere ("background field")

$\triangle A A A$ ANAD
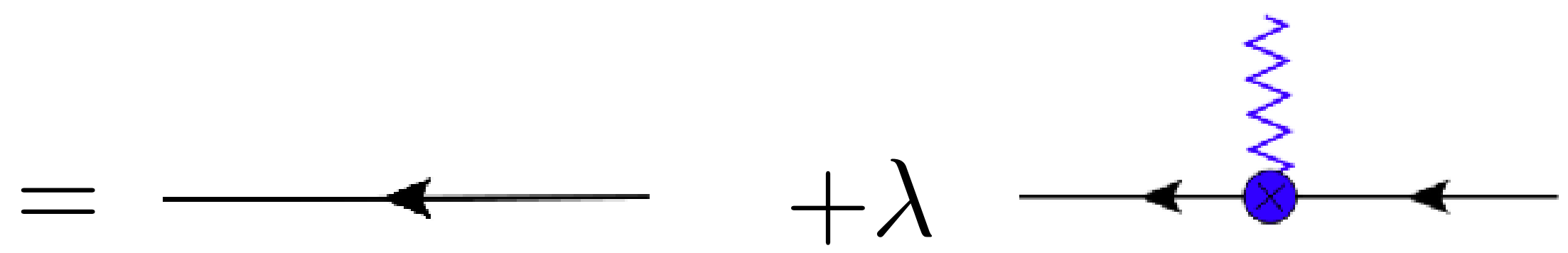

Correlation functions encode linear (quadratic, ...) response to background field in linear (quadratic, ...) terms of polynomial

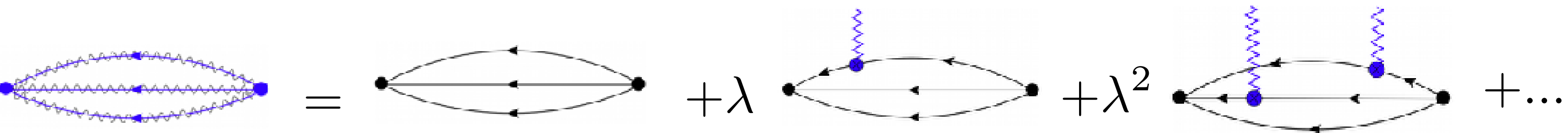

Nuclear correlation functions containing current insertions can be formed as straightforwardly as two-point functions
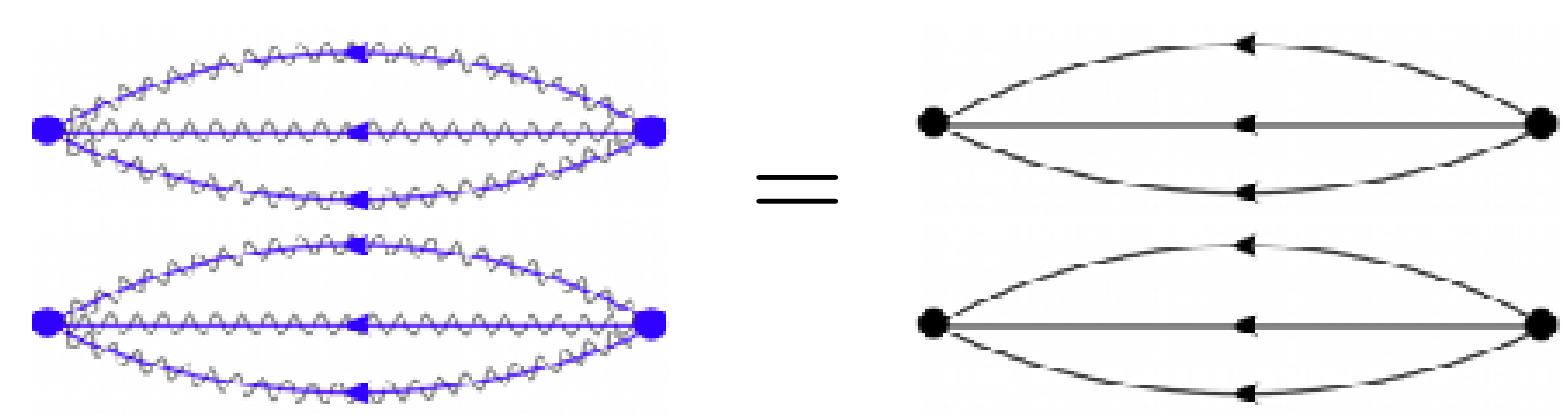

Savage, MW et al [NPLQCD], PRL 119 (2017)

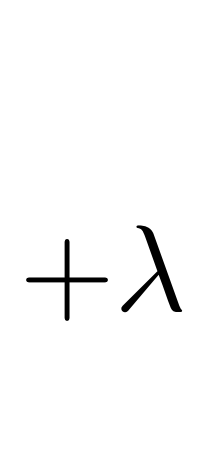

Tiburzi, MW et al [NPLQCD], PRD 96 (2017) 


\section{Extracting matrix elements}

Correlation functions exact polynomials in background field strength

Current matrix elements obtained from linear term

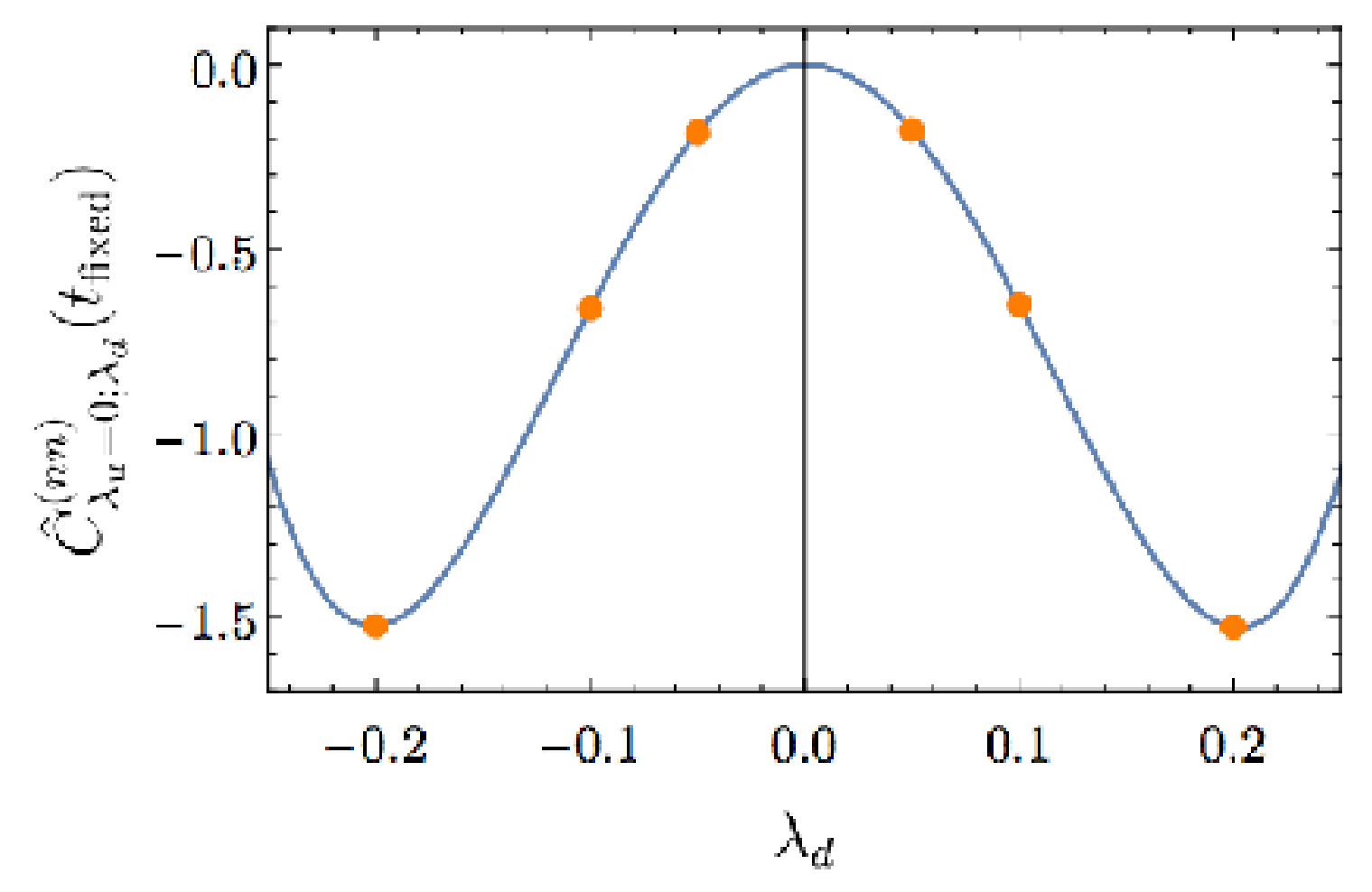

$$
C_{A}^{(\lambda)}(t)=<A(t) A^{\dagger}(0)>+\lambda \sum_{y}\left\langle A(t) J(y) A^{\dagger}(0)\right\rangle+O\left(\lambda^{2}\right)
$$

Inserting complete sets of states gives Euclidean spectral representation

$$
\left.C_{A}^{(\lambda)}(t)\right|_{O(\lambda)}=\left|Z_{0}\right|^{2} e^{-E_{0} t}\left[c+t\langle A|J| A\rangle+O\left(e^{-\delta t}\right)\right]
$$

Fit to LQCD

Fixed-order background field formalism also helps organize EFT matching 


\section{Neutron $\beta$-decay}

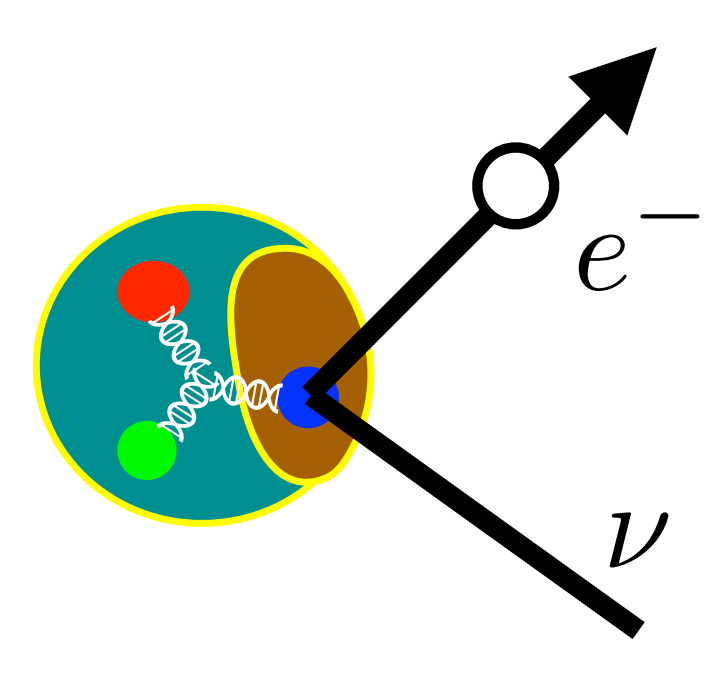

Neutron decay rate governed by axial charge

$$
\begin{gathered}
g_{A}=\left\langle p\left|A_{3}^{+}\right| n\right\rangle=\left\langle p\left|A_{3}^{3}\right| p\right\rangle \\
A_{\mu}^{a}=A_{\mu}^{a}(\mathbf{q}=0)=\int_{\mathbf{y}} \bar{q}(\mathbf{y}) \gamma_{\mu} \gamma_{5} \tau^{a} q(\mathbf{y})
\end{gathered}
$$

Isospin limit

$g_{A}$ obtained from nucleon background field correlation function

$\partial_{t}\left(\frac{\left.C_{p}^{\left(\lambda_{u}-\lambda_{d}\right)}(t)\right|_{O(\lambda)}}{C_{p}(t)}\right)=\left\langle p\left|A_{3}^{3}\right| p\right\rangle+O\left(e^{-\delta t}\right)$

Axial current renormalization $Z_{A}$ computed separately

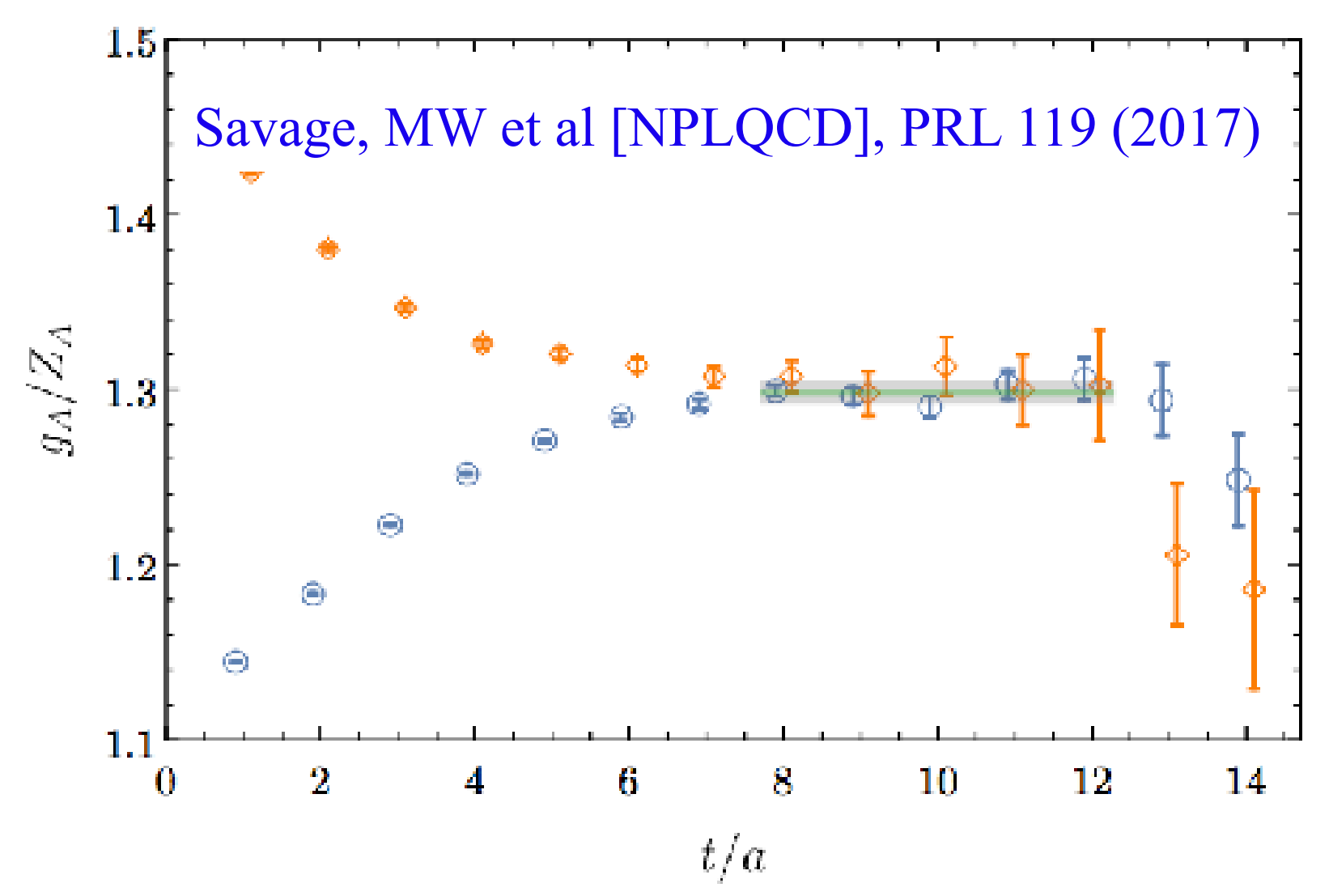

Accurate LQCD calculations at physical quark masses performed by

several groups, reviewed by FLAG: Aoki et al [FLAG], arXiv:1902.08191 


\section{Proton-proton fusion}

Combining lattice QCD and EFT allows more complex reactions to be studied

LQCD/EFT matching for two nucleon correlation functions in a box with a background axial field:

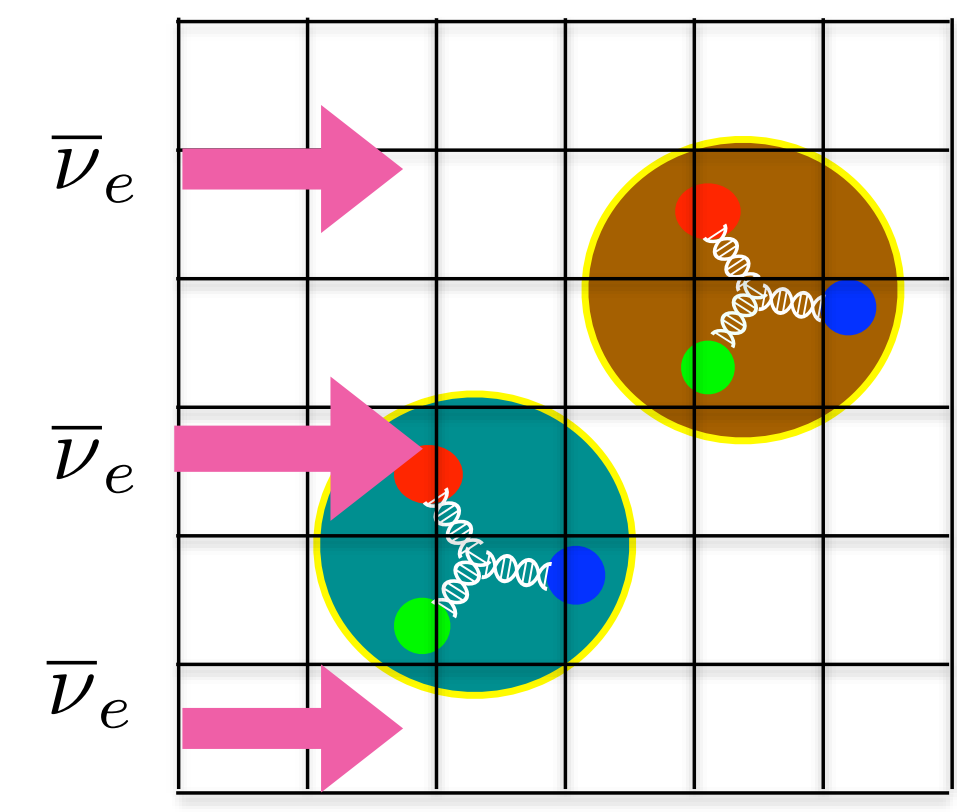

$$
i C_{p p \rightarrow n p\left({ }^{3} S_{1}\right)}=\mathscr{M}_{p p \rightarrow n p\left({ }^{3} S_{1}\right)}=g A(1+S)-L_{1 A}
$$

Used for LQCD determination of two-body currents in proton-proton fusion

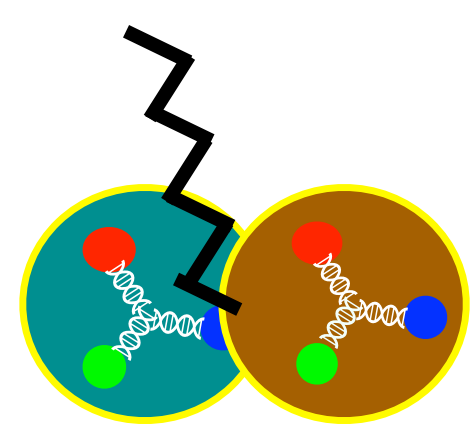

$L_{1 A}=3.9(0.2)(1.0)(0.4)(0.9) \mathrm{fm}^{3}$

Savage, MW et al [NPLQCD], PRL 119 (2017)

\begin{tabular}{lcc}
\hline & method & $L_{1 A}\left(\mathrm{fm}^{3}\right)$ \\
\hline two-body & reactor $\bar{\nu}+d$ & $3.6 \pm 5.5[11]$ \\
& ES, CC, NC in SNO & $4.0 \pm 6.3[41]$ \\
& MuSun proposal & \pm 1.25 \\
\hline \hline three-body & tritium beta decay & $4.2 \pm 3.7[11], 4.2 \pm 0.1[41]$ \\
\hline other & helioseismology & $4.8 \pm 6.7[42]$ \\
\hline
\end{tabular}

Andreev et al (MuSun) arXiv 1004.1754 


\section{Tritium $\beta$-decay}

Triton decay rate governed by Gamow-Teller matrix element

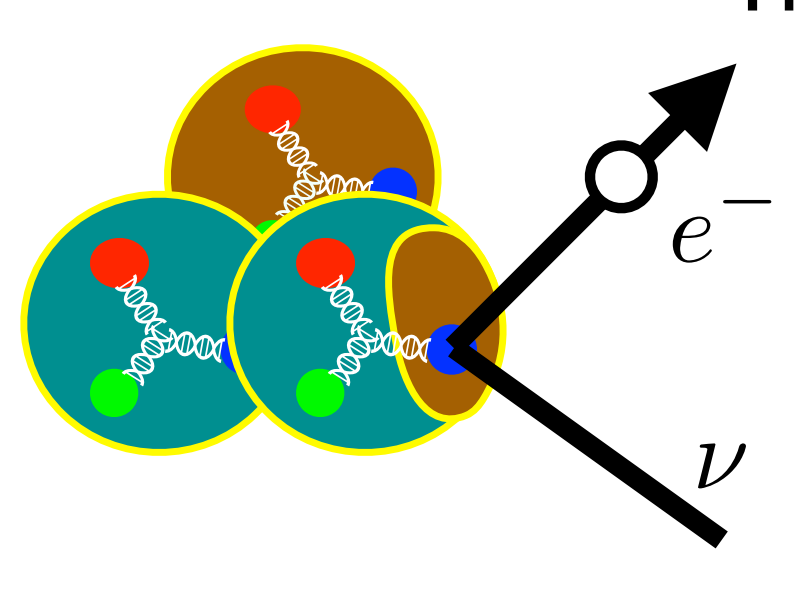

Computed in ChEFT

$$
g_{A}\left({ }^{3} \mathrm{H}\right)=\left|\left\langle{ }^{3} \mathrm{He}\left|A_{3}^{+}\right|{ }^{3} \mathrm{H}\right\rangle\right|=\left|\left\langle{ }^{3} \mathrm{H}\left|A_{3}^{+}\right|{ }^{3} \mathrm{H}\right\rangle\right|
$$

Baroni et al, PRC 98 (2018)

After fitting LECs to experimental triton $\beta$ decay rate predicts

$$
\frac{\left|\left\langle{ }^{3} \mathrm{He}\left|A_{3}^{+}\right|{ }^{3} \mathrm{H}\right\rangle\right|}{g_{A}}=0.951(13)
$$

Deviations from 1 arise from two-body currents and multi-nucleon interactions

NLO calculations in pionless EFT relate nuclear effects to the two-body axial current coupling $L_{1 A}$ appearing in proton-proton fusion 


\section{Tritium $\beta$-decay, $m_{\pi} \sim 806 \mathbf{M e V}$}

With fixed-order axial background fields, triton GT matrix element calculation analogous to proton axial charge

$$
\partial_{t}\left(\frac{\left.C_{3_{\mathrm{He}}^{\left(\lambda_{u}-\lambda_{d}\right)}(t)}\right|_{O(\lambda)}}{C_{{ }^{3} \mathrm{He}}(t)}\right)=\left\langle{ }^{3} \mathrm{He}\left|A_{3}^{3}\right|^{3} \mathrm{He}\right\rangle+O\left(e^{-\delta t}\right)
$$

Renormalization factors cancel from isovector matrix element ratios, precise results obtained for ratio

$$
\frac{\left|\left\langle{ }^{3} \mathrm{He}\left|A_{3}^{+}\right|{ }^{3} \mathrm{H}\right\rangle\right|}{g_{A}}=0.987(4)
$$

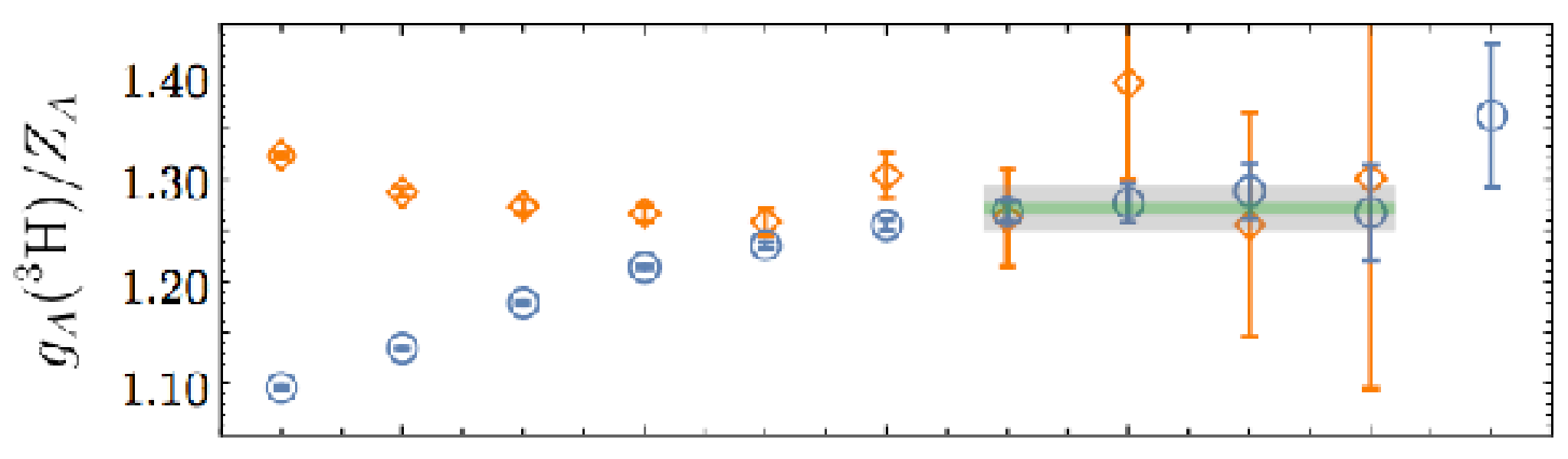

$N_{f}=3, m_{\pi}=806(9) \mathrm{MeV}, a=0.145(2) \mathrm{fm}$

Savage et al [NPLQCD], PRL 119 (2017) 


\section{Going to lighter quark masses}

Much higher statistics needed at lighter quark masses

$$
\begin{array}{ll}
m_{\pi} \sim 806 \mathrm{MeV} & m_{\pi} \sim 450 \mathrm{MeV} \\
N_{s r c}=10,592 & N_{s r c}=281,472
\end{array}
$$

"Autofitter" algorithm

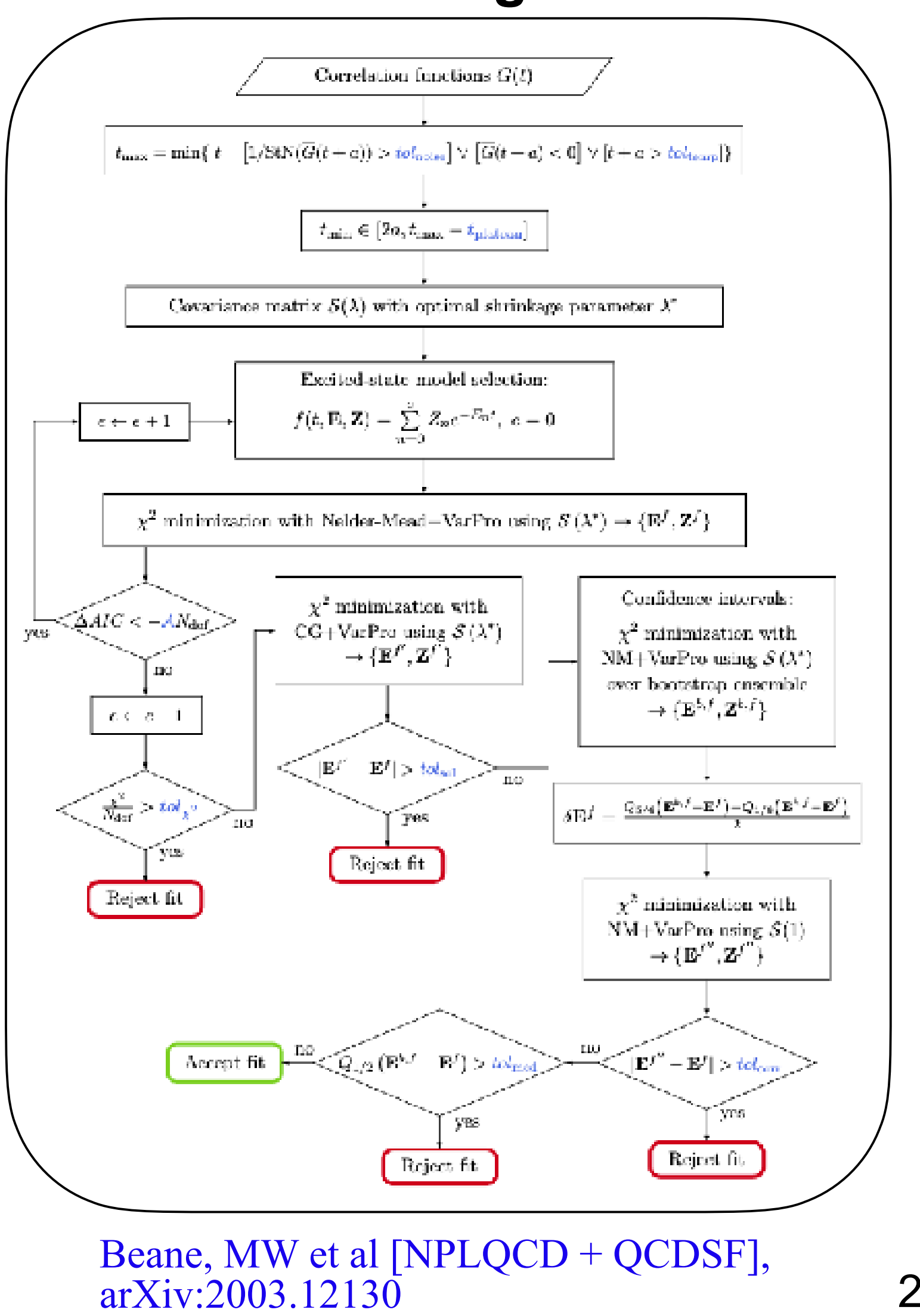

$$
\mathrm{StN} \approx \frac{1}{\sqrt{N_{s r c}}} e^{-A\left(M_{N}-\frac{3}{2} m_{\pi}\right) t}
$$

Faster noise growth makes identifying "plateau" challenging

Automated sampling of fitting choices essential for reliable results 


\section{The triton at $m_{\pi} \sim 450 \mathrm{MeV}$}

New calculations of ${ }^{3} \mathrm{H}$ performed with $m_{\pi} \sim 450 \mathrm{MeV}$

[NPLQCD], arXiv:2004.XXXX
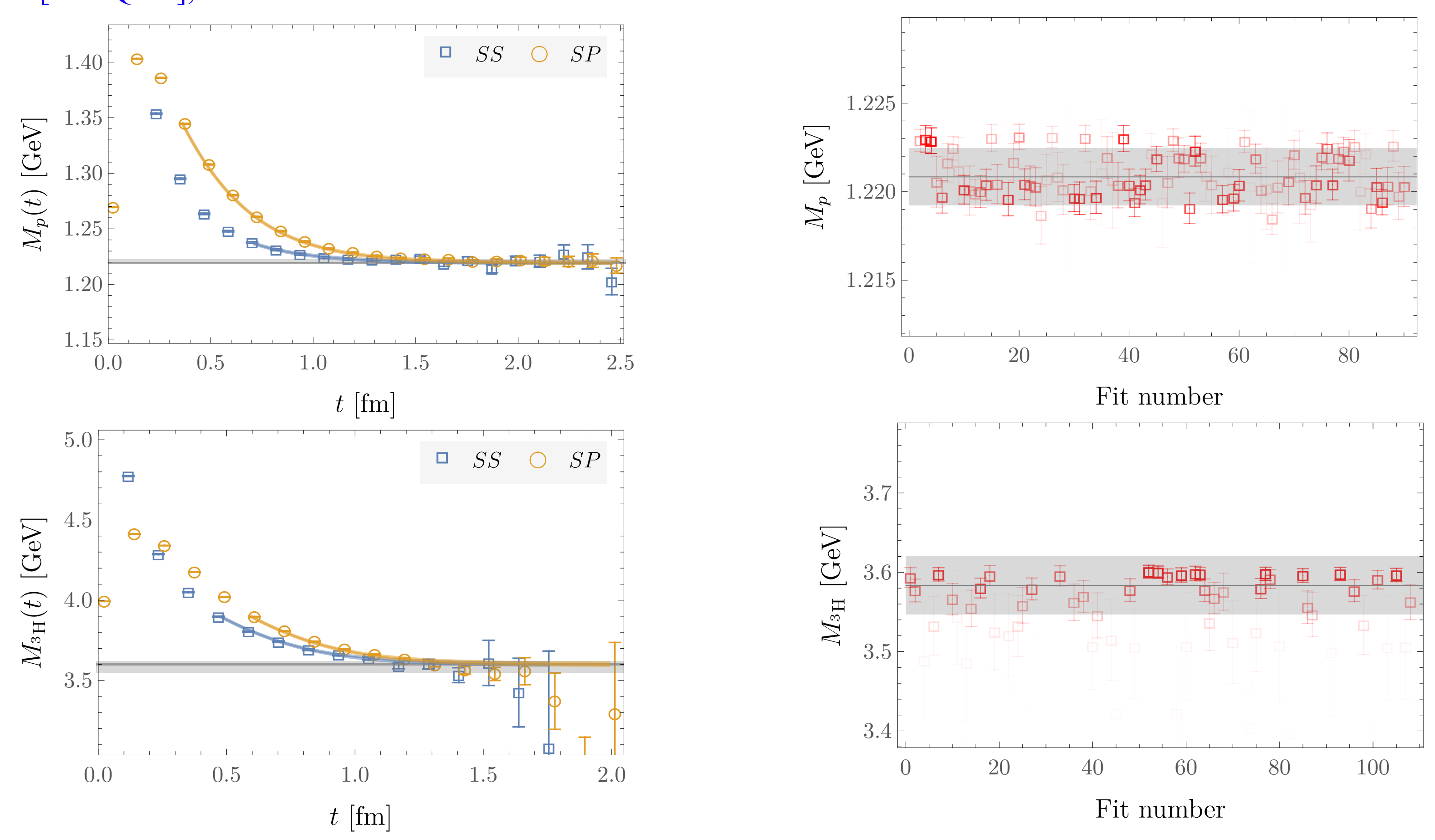

All possible fit ranges sampled, multi-state fit model selected for each, weighted average taken of all fits passing various reliability checks 


\section{Triton binding vs quark mass}

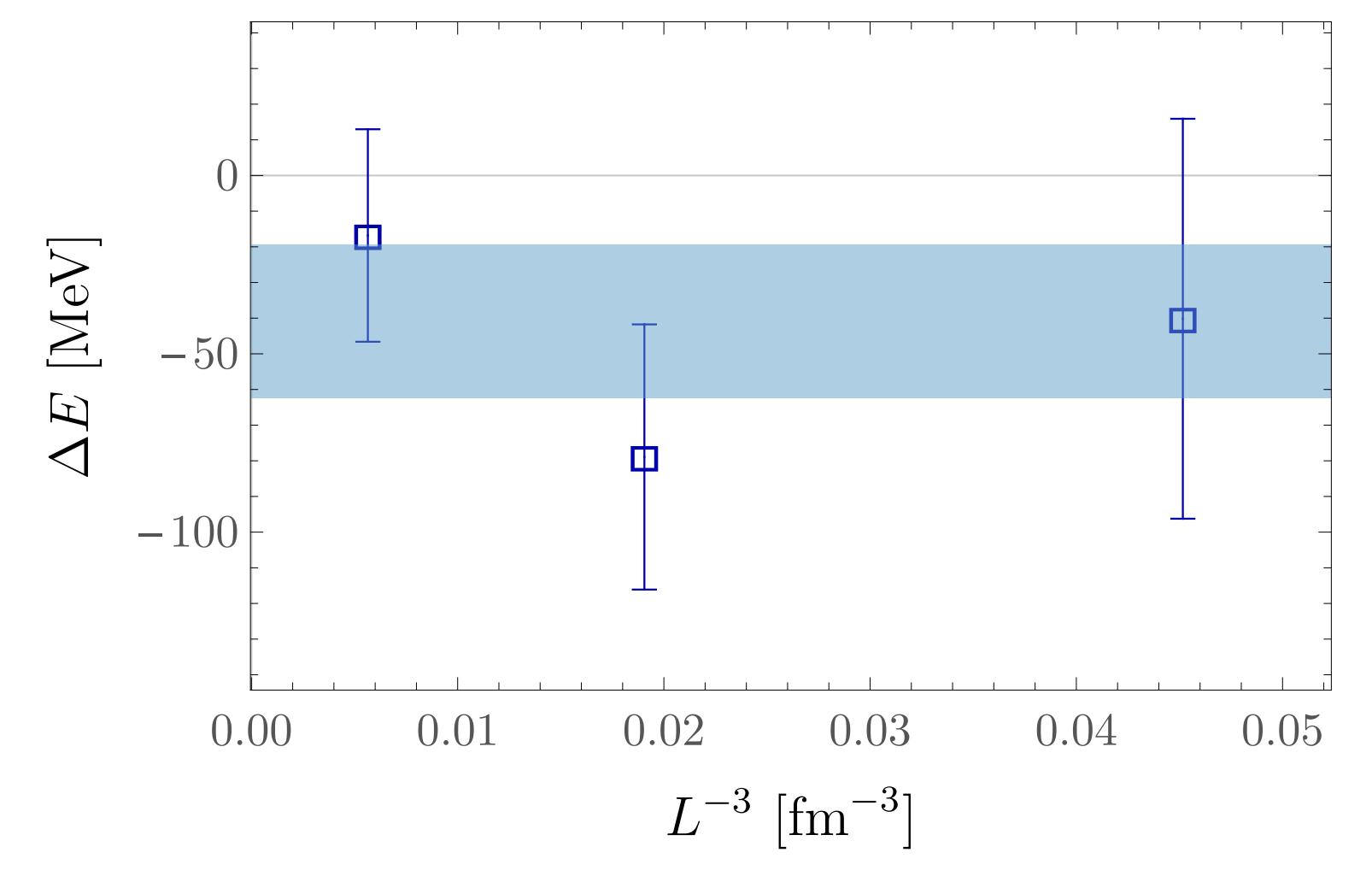

Volume dependence of triton energy shift consistent with bound state

Pion mass dependence appears consistent between different LQCD results

Simple extrapolations to physical point imprecise but consistent with experimental results

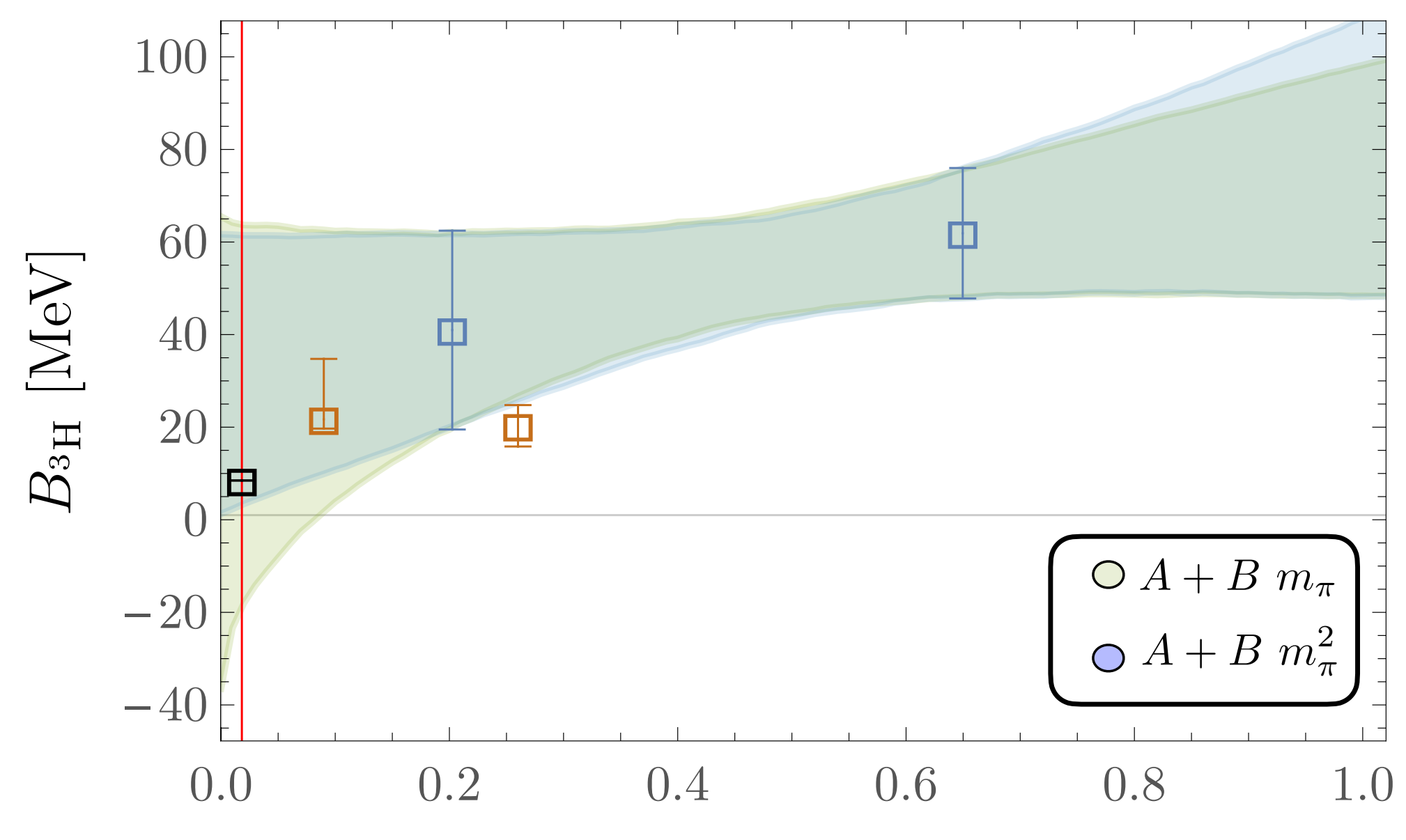

$m_{\pi}^{2}\left[\mathrm{GeV}^{2}\right]$ 


\section{Axial currents at $m_{\pi} \sim 450 \mathrm{MeV}$}

Same fitting procedure applied to correlation functions with background axial fields in order to extract matrix elements
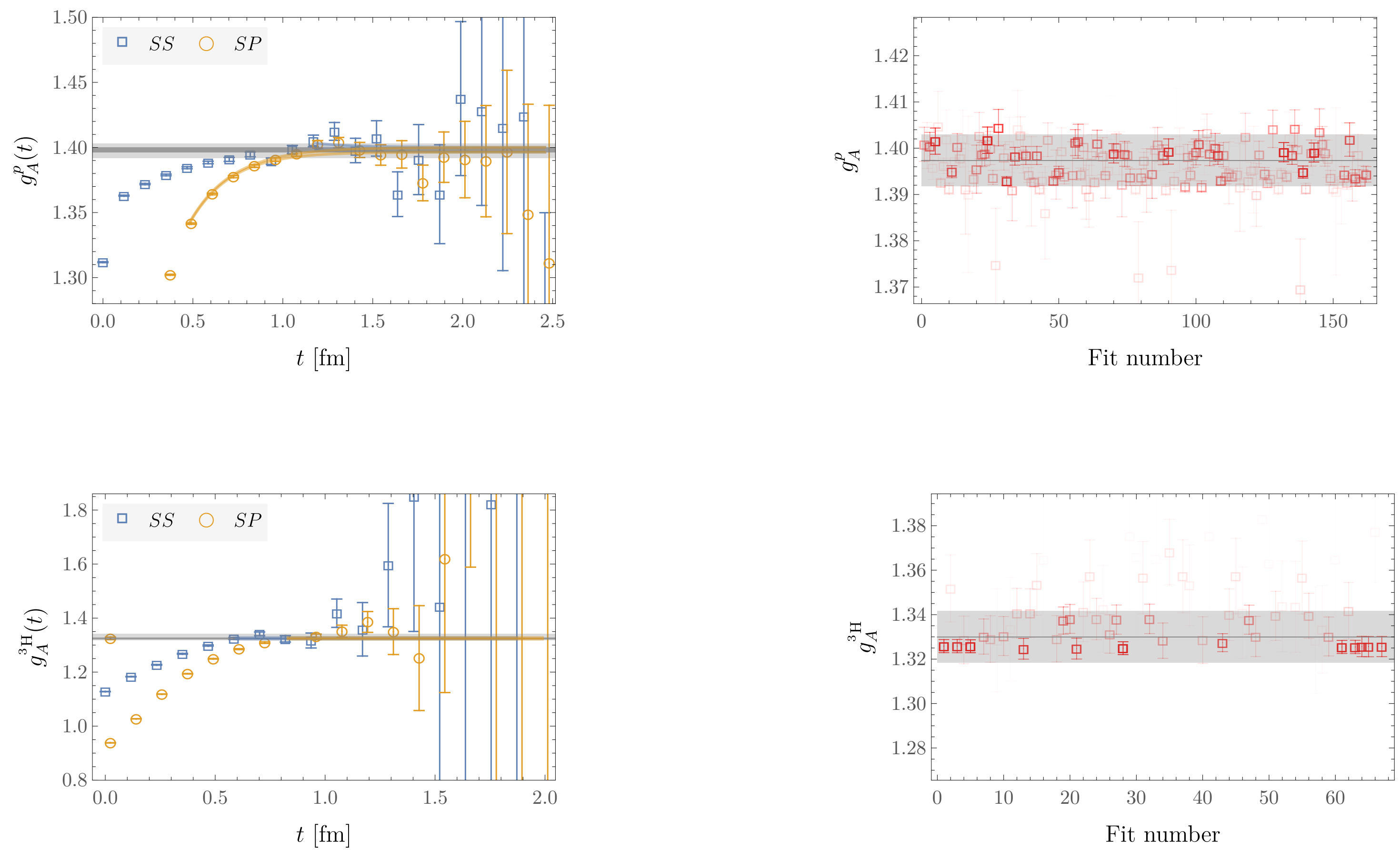


\section{Axial charge vs quark mass}

Simple extrapolations of proton and ${ }^{3} \mathrm{H}$ axial charges similarly consistent with physical results
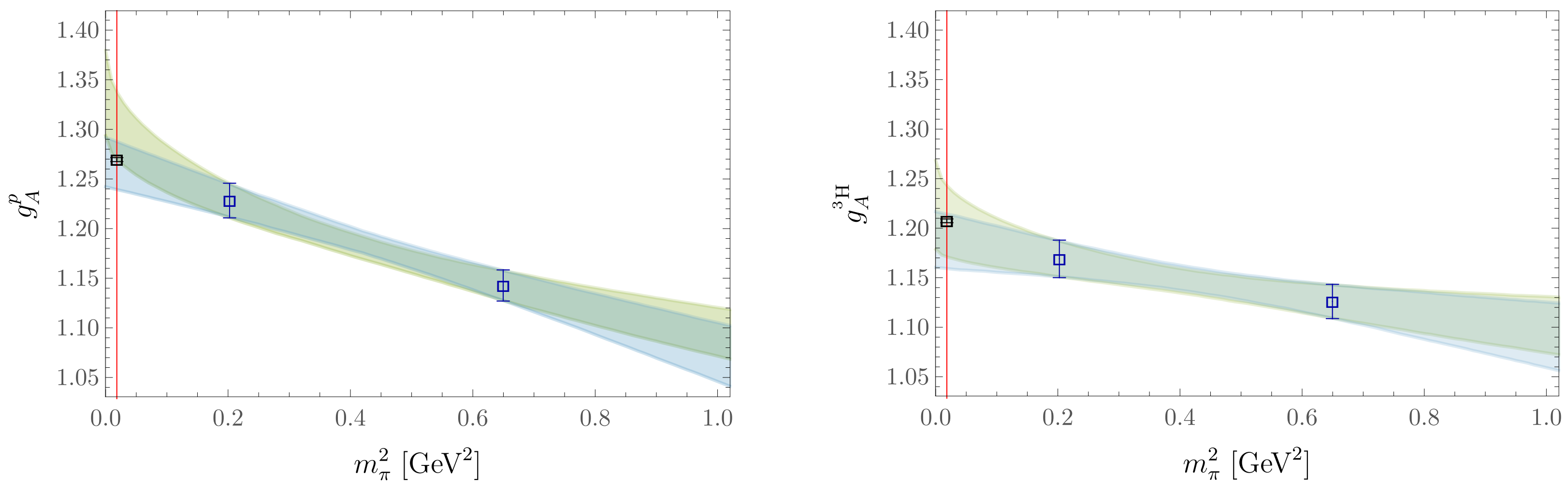

(Note many available LQCD results for the proton are not shown) 


\section{Nuclear effects on axial structure}

Ratios of ${ }^{3} \mathrm{H}$ and proton

axial charges determined more precisely than individual charges (renormalization cancels)

$$
\begin{gathered}
m_{\pi} \sim 450 \mathrm{MeV} \\
\frac{g_{A}^{{ }^{3} \mathrm{H}}}{g_{A}^{p}}=0.952(9)
\end{gathered}
$$

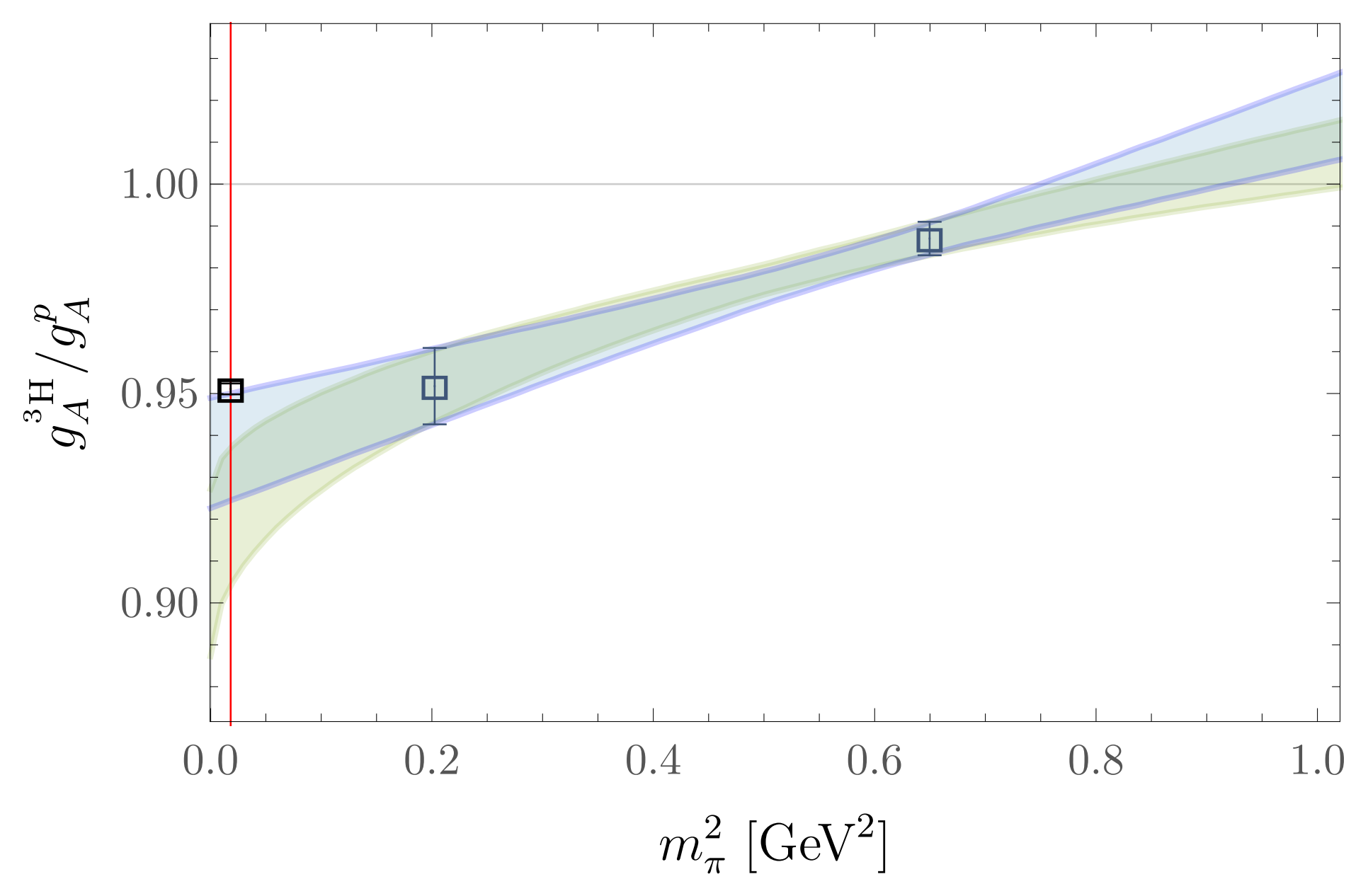

Results at $m_{\pi} \sim 450 \mathrm{MeV}$ and simple extrapolations consistent with physical results

Nuclear effects on axial structure more significant at lighter pion mass

- are chiral dynamics important for emergence of nuclear effects on axial structure? 


\section{Flavor dependence}

Beyond validation, LQCD can compute properties of electroweak reactions that are difficult to access experimentally, e.g. flavor dependence

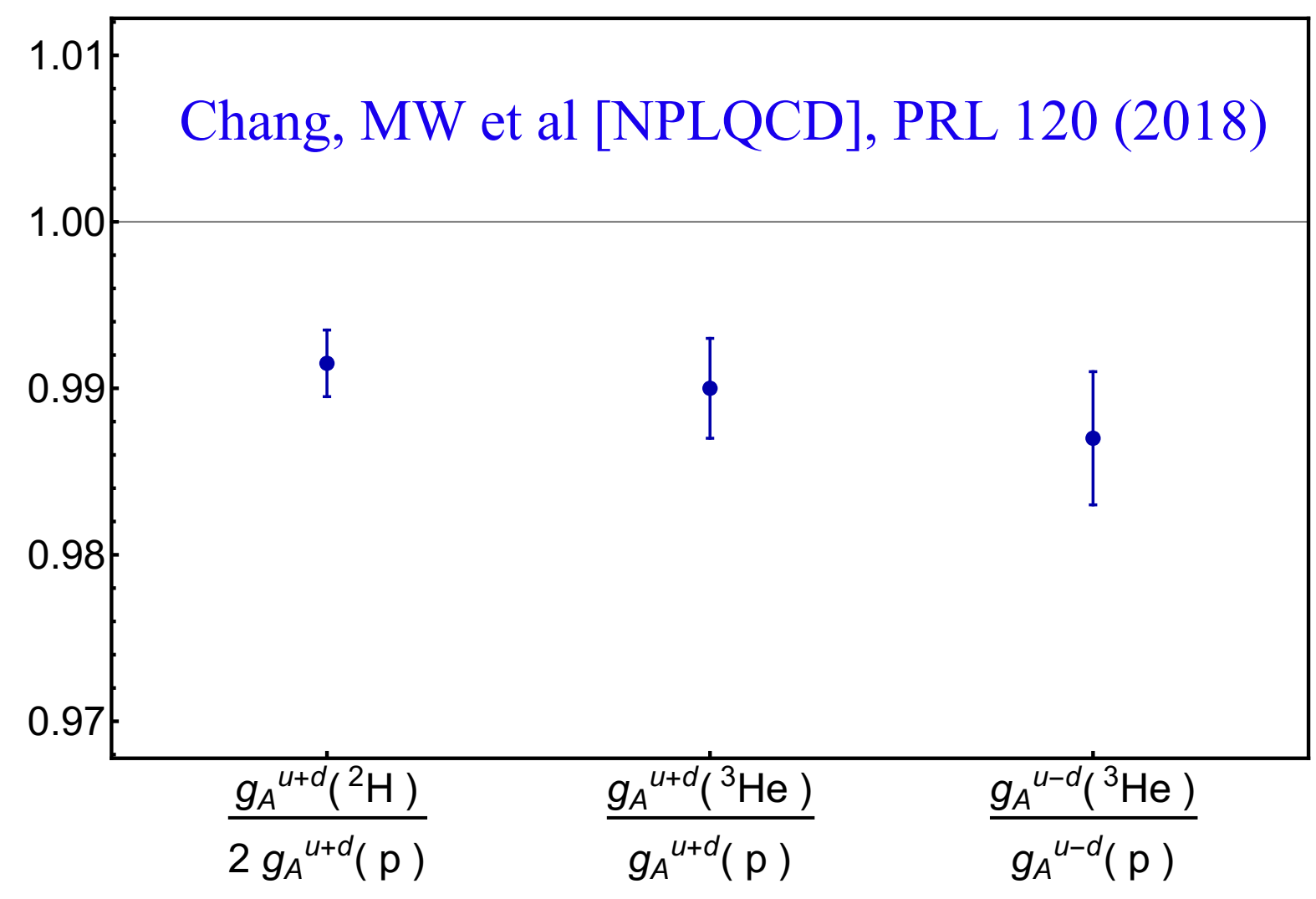

Nuclear modifications of $u$ and $d$ quark axial couplings $\mathrm{O}(1 \%)$ for $\mathrm{A}=2-3$ nuclei, $m_{\pi} \sim 806 \mathrm{MeV}$

$N_{f}=3, m_{\pi}=806(9) \mathrm{MeV}, a=0.145(2) \mathrm{fm}$

Flavor-singlet matrix elements needed for neutral weak interactions, understanding quark/gluon spin structure of nuclei

$$
g_{A, h}^{(u+d+s)}=\left\langle h, \mathbf{p}, \lambda\left|\bar{q} \gamma_{\mu} \gamma_{5} q\right| h, \mathbf{p}, \lambda\right\rangle=s_{\mu}^{h} \int_{0}^{1} d x \Delta q_{h}^{i}(x)
$$

Extensions to include momentum transfer and/or derivative operators will constrain axial form factors and structure functions 


\section{Beyond the Standard Model currents}

LQCD can also compute nuclear reactions induced by BSM currents, needed to interpret e.g. dark matter direct detection experiments

Larger nuclear modifications of scalar than axial structure at $m_{\pi} \sim 806 \mathrm{MeV}$

Extensions to physical quark masses will provide inputs to EFTs for dark matter direct detection, e.g. Fieguth et al, PRD 97 (2018) Hoferichter et al, PRD 99 (2019)
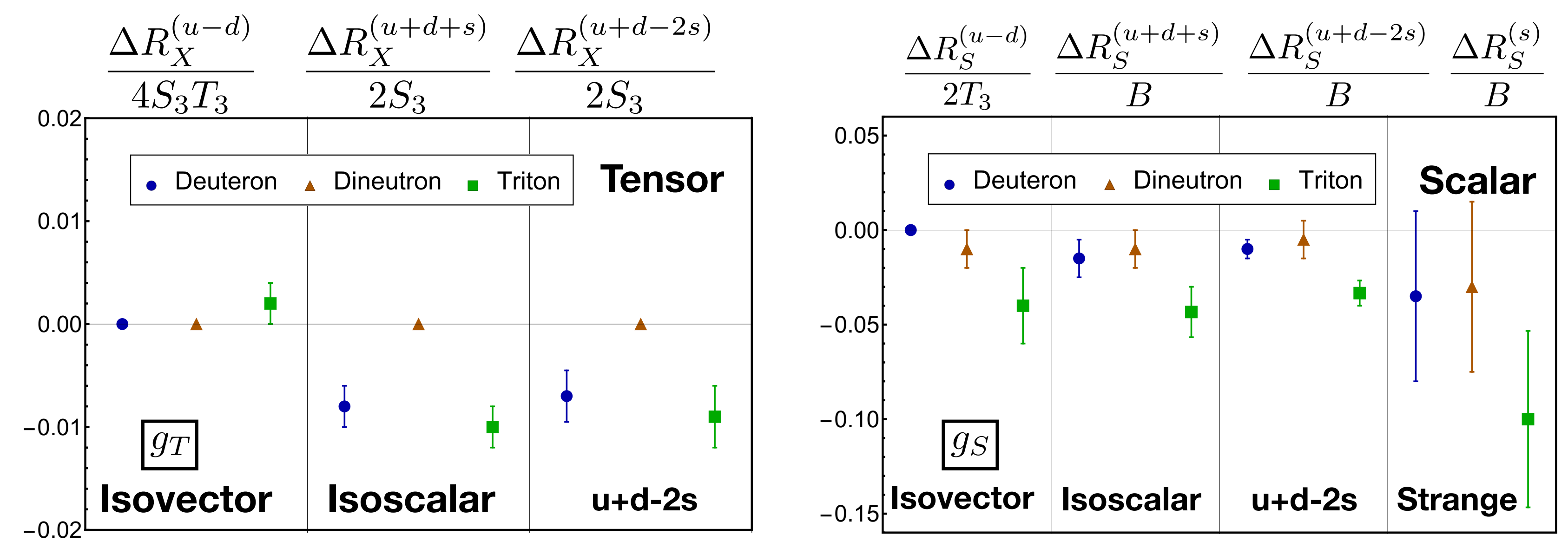

Chang, MW et al [NPLQCD], PRL 120 (2018) 


\section{Towards larger nuclei}

LQCD results in the few-nucleon sector can be used to tune EFTs that can efficiently predict properties of larger nuclei

Provides near-term strategy for QCD-based predictions of the properties of larger nuclei

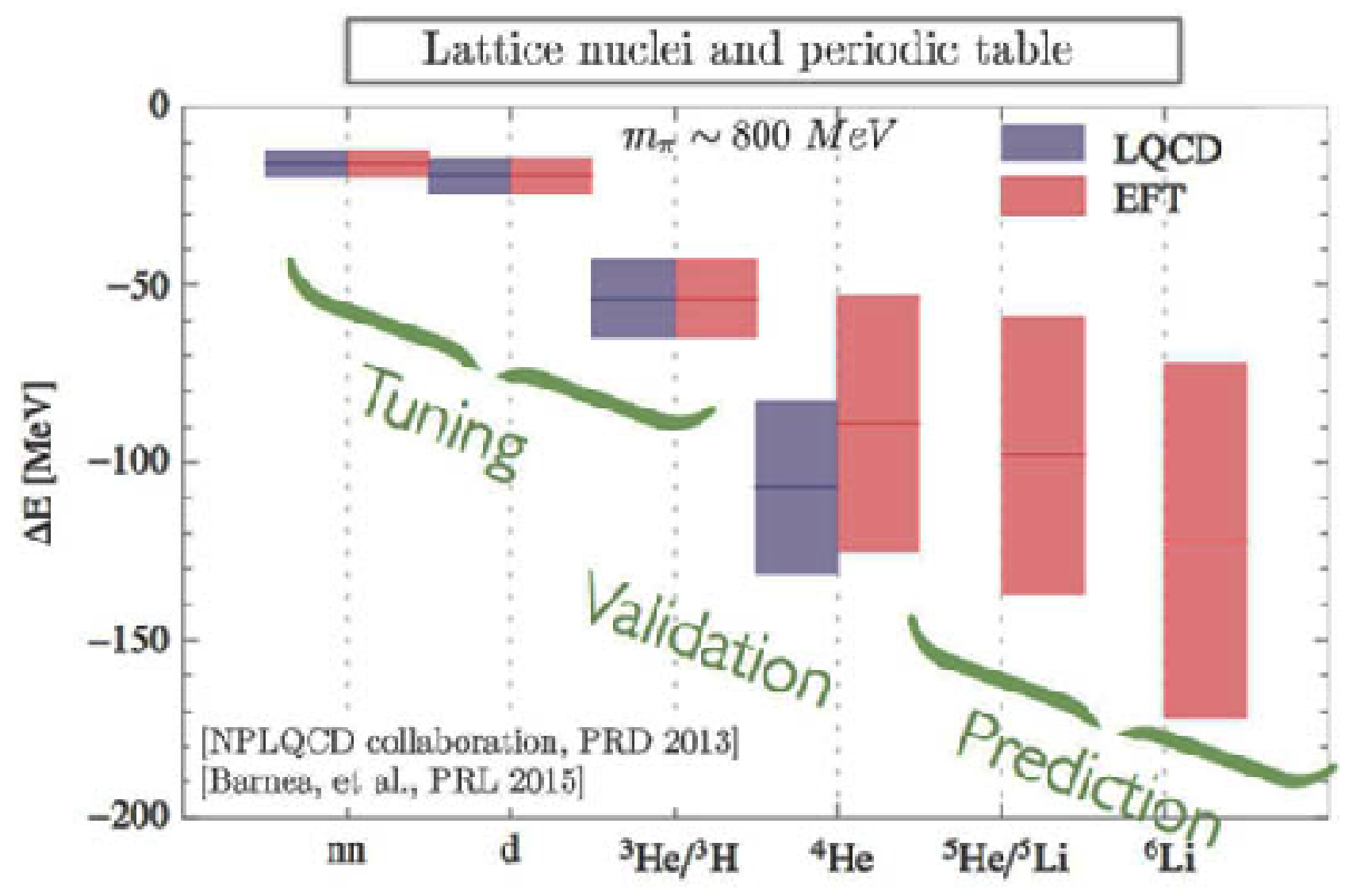




\section{Towards larger nuclei}

LQCD results in the few-nucleon sector can be used to tune EFTs that can efficiently predict properties of larger nuclei

Provides near-term strategy for QCD-based predictions of the properties of larger nuclei

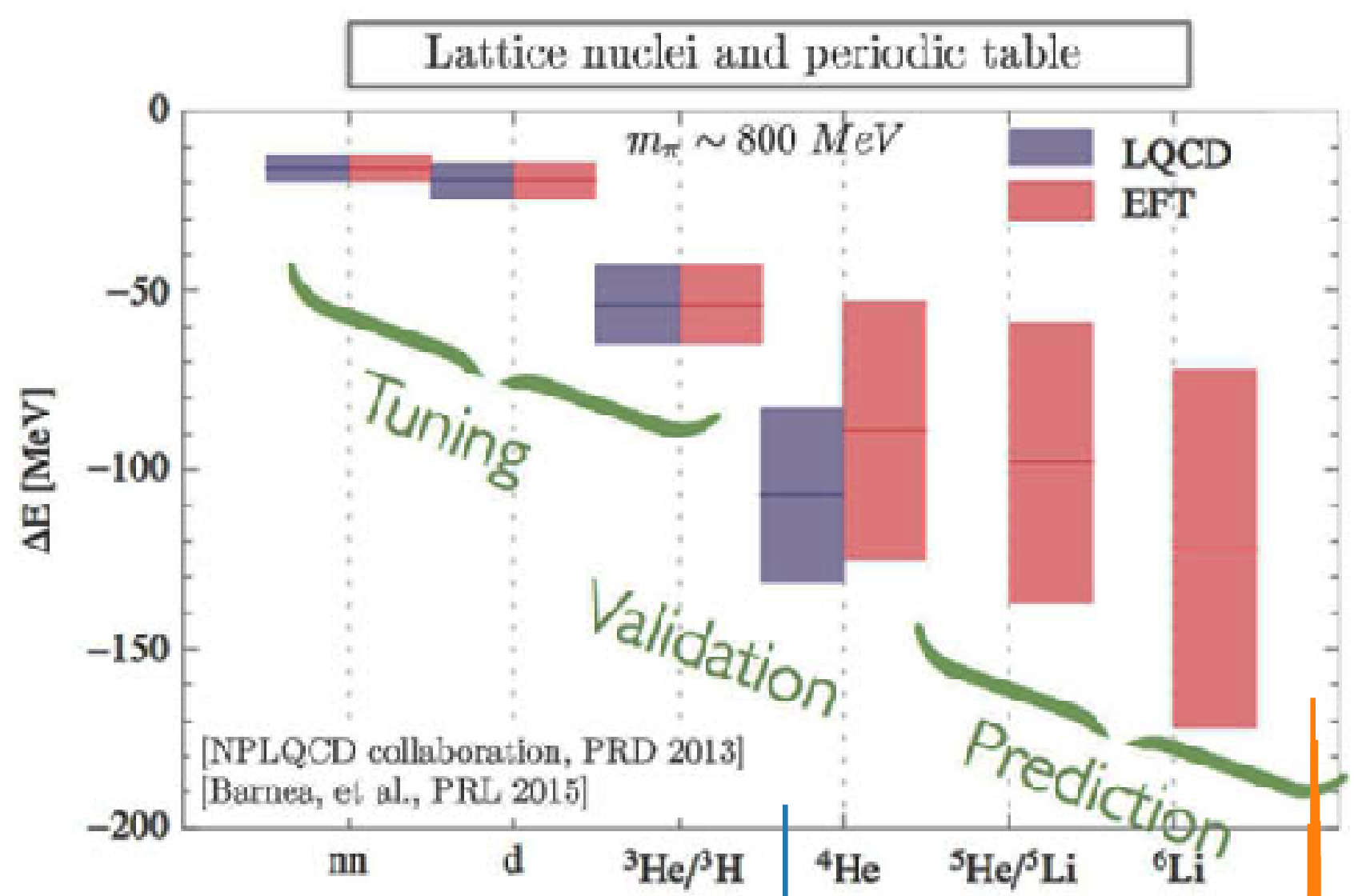

Techniques to mitigate the signal-to-noise problem could eventually allow exponentially more efficient LQCD calculations of nuclei

Recent progress on signal-to-noise problems in 2D U(1) gauge theory achieved by path integral contour deformations

Detmold, Kanwar, MW, Warrington, arXiv:2003.05914

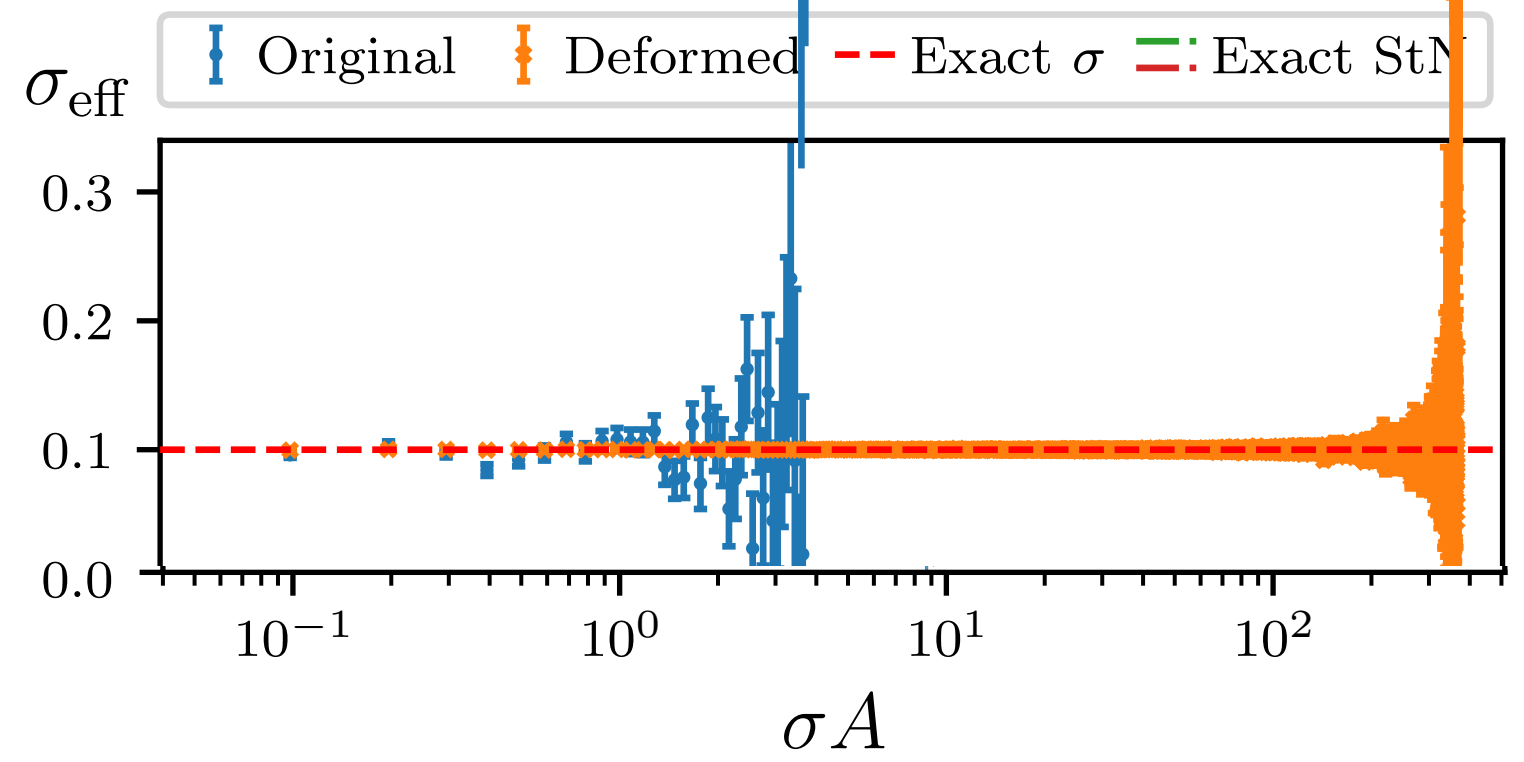




\section{From quarks to nuclei}

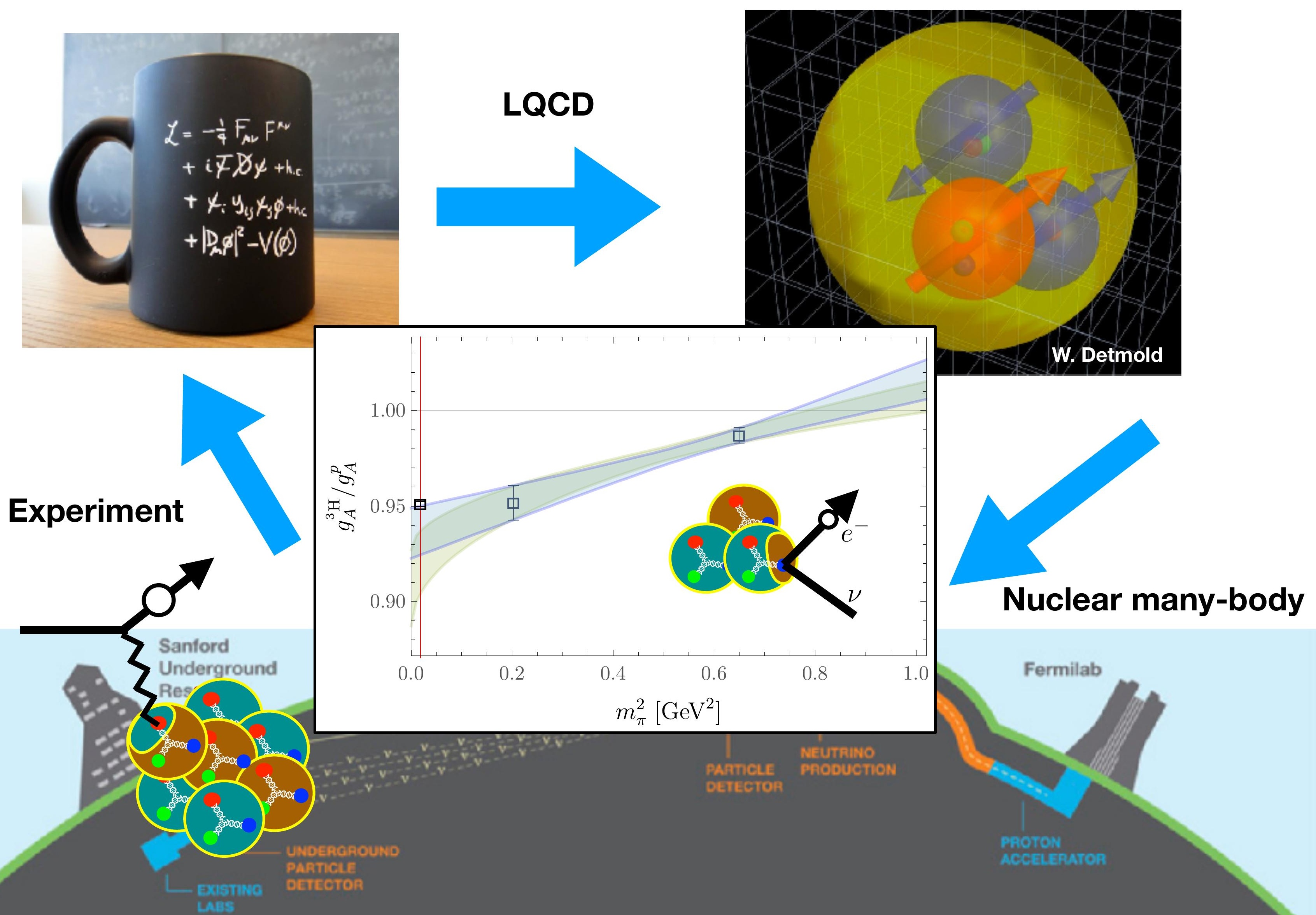

